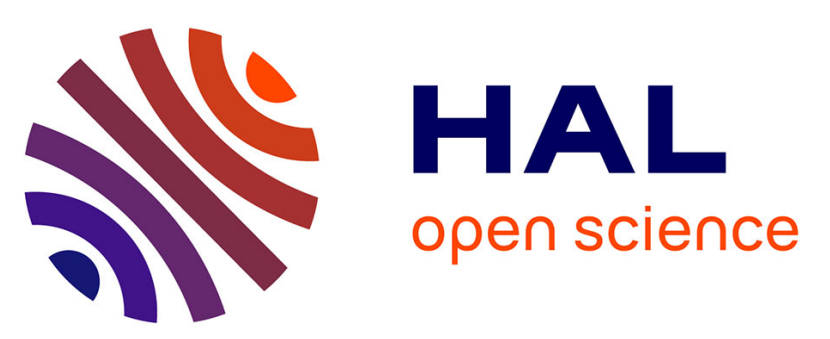

\title{
A One-Pot Three-Step Synthesis of Z-Trisubstituted Olefins from Arylalkynes and Their Cyclization into 4-Aryl-2H-chromenes
}

Evelia Rasolofonjatovo, Bret Tréguier, Olivier Provot, Abdallah Hamze, Jean-Daniel Brion, Mouad Alami

\section{To cite this version:}

Evelia Rasolofonjatovo, Bret Tréguier, Olivier Provot, Abdallah Hamze, Jean-Daniel Brion, et al.. A One-Pot Three-Step Synthesis of Z-Trisubstituted Olefins from Arylalkynes and Their Cyclization into 4-Aryl-2H-chromenes. European Journal of Organic Chemistry, 2012, 2012 (8), pp.1603-1615. 10.1002/ejoc.201101735 . hal-02394410

\section{HAL Id: hal-02394410 https://hal.science/hal-02394410}

Submitted on 4 Dec 2019

HAL is a multi-disciplinary open access archive for the deposit and dissemination of scientific research documents, whether they are published or not. The documents may come from teaching and research institutions in France or abroad, or from public or private research centers.
L'archive ouverte pluridisciplinaire HAL, est destinée au dépôt et à la diffusion de documents scientifiques de niveau recherche, publiés ou non, émanant des établissements d'enseignement et de recherche français ou étrangers, des laboratoires publics ou privés. 


\title{
A one pot three-steps synthesis of $Z$-trisubstituted olefins from arylalkynes and their cyclization into 4-aryl-2H-chromenes
}

\author{
Evelia Rasolofonjatovo,[a] Bret Tréguier,[a] Olivier Provot,[a]* Abdallah Hamze,[a] Jean-Daniel \\ Brion[a] and Mouâd Alami*[a]
}

Keywords: Alkyne hydration/ Olefin / Chromene / N-tosylhydrazone/ Palladium

A rapid and versatile access from arylalkynes $\mathbf{3}$ to $Z$-trisubstituted olefins $\mathbf{2}$ and their cyclization into 4 -aryl- $2 H$-chromenes $\mathbf{1}$ is described. In a one-pot fashion, alkynes $\mathbf{3}$ were first hydrated, then transformed into $N$-tosylhydrazones and finally coupled with ortho substituted aryl halides under palladium catalysis to give trisubstituted olefins 2 in good yields and very high to total $Z$-selectivity.
1,1-Diarylolefins 2 having an ortho-OMOM substituent underwent rapid cyclization in the presence of PTSA to give a variety of 4-aryl$2 H$-chromenes in good to excellent yields. This methodology was also successfully applied to prepare 5-aryl-2,3-dihydrobenzo[b] oxepine $\mathbf{4}$ from the required arylbutynol $\mathbf{3 b}$. [a] Univ Paris-Sud, CNRS, BioCIS UMR 8076, Laboratoire de Chimie Thérapeutique, LabEx LERMIT, Faculté de Pharmacie, rue J.-B. Clément, Châtenay-Malabry F-92296, France

Fax: +33-1 46.83.58.28

E-mail: olivier.provot@u-psud.fr or mouad.alami@u-psud.fr

\section{Introduction}

Chromenes ${ }^{[1]}(2 H$-benzopyran derivatives $)$ are an important @ss of natural and synthetic compounds that exhibit a broad range of pharmacological properties. ${ }^{[2]}$ Among them, 4-arylchromenes, also named neoflavenes, is one of the most important subfamilies of chromenes, whose derivatives show promising biological activities, including retinoic acid receptor $\alpha$-selective antagonists, ${ }^{[3]}$ and potassium channel activators, with smoothmuscle relaxant activity in a variety of cardiovascular and bronchopulmonary diseases. ${ }^{[4]}$ This wide range of biological activity displayed by these heteroaromatic compounds has made them popular synthetic targets.

Figure 1. Structure of $\mathrm{CA}-4$, isoCA-4 and synthetic tubulin assembly inhibitors $\mathbf{1}$ and $\mathbf{2}$.

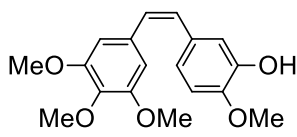

CA-4<smiles>[R]c1ccccc1C(=CCOCC)c1ccccc1</smiles><smiles>C=C(c1ccc(OC)c(O)c1)c1cc(OC)c(OC)c(OC)c1</smiles>

isoCA-4<smiles>[R]c1ccc(C2=CCOc3c([R])cccc32)cc1</smiles>

In an ongoing project aimed at developing novel antimitotic substances ${ }^{[5]}$ we reported that the bioisoteric replacement of the 1,2-ethylene bridge of CA- 4 by a 1,1-ethylene linker resulted in stable and non-isomerizable compounds (e.g.; isoCA-4), displaying biological activities comparable to that of the natural product. ${ }^{[5 b, c]}$ As an outcome of the exhaustive structure-activity relationships studies of isoCA-4, we were interested in developing a novel methodology that provides a rapid and efficient access to 4-aryl$2 \mathrm{H}$-chromenes $\mathbf{1}$, regarded as constraint analogues of isoCA-4. A survey of the literature showed that few routes have been developed for the preparation of 4-aryl- $2 \mathrm{H}$-chromenes, including the coupling of 4-OTf-chromene derivatives with arylboronic acids, ${ }^{[6]}$ a reaction of 4-methoxycarbonylchromane-3-one with aryllead tri-acetates, ${ }^{[7]}$ ring-closing metathesis ${ }^{[8]}$ and so on. ${ }^{[9]}$

Herein, we disclose the full details of our strategy towards the synthesis of 4-aryl-2H-chromenes $\mathbf{1}$, which involves the cyclization of a new series of 1,1-diarylethylene derivatives $2 .^{[10]}$ These later having a trisubstituted double bond, constitute another interesting class of isoCA-4 analogues, potentially endowed with biological activities ${ }^{[11]}$

\section{Results and Discussion}

As shown in Table 1, alkynol 3a ${ }^{[12]}$ was first hydrated in EtOH using a catalytic amount of $p$-toluensulfonic acid (PTSA) ${ }^{[13]}$ Under this environmentally friendly procedure, the resulting ketone was transformed into its $\mathrm{N}$-tosylhydrazone, and then coupled with 4iodoanisole under palladium catalysis $\left(\mathrm{PdCl}_{2}(\mathrm{MeCN})_{2} / \mathrm{Xphos}\right)^{[14,5 \mathrm{c}]}$ to give trisubstituted olefin $\mathbf{2 a}$ in a good overall yield (63\%, entry 1). It should be noted that this three-step synthesis of 2a from alkynol 3a was carried out without any purification of the intermediates generated in each stage.

Interestingly, we found that the position of the substituent $\mathrm{R}$ at the aromatic ring of aryl halides appears to play a crucial role for the reaction stereochemistry. Thus, when the Pd-coupling step was performed with meta aryl halide derivatives, such as 3-iodoanisole or 2-bromo-6-methoxynaphtalene, the reaction furnished trisubstituted olefins $\mathbf{2 b}$ and $\mathbf{2 c}$, respectively, with a slight $Z$ stereoselectivity (entries 2, 3). More interestingly, this $Z$ selectivity ${ }^{[15]}$ was even more remarkable when ortho iodoanisole was used in the Pd-coupling step, furnishing olefin $\mathbf{2 d}$ in a good overall yield and a high diastereoisomeric ratio (77\%, Z/E 90/10, entry 4). Examples depicted in entries 5-9 confirm that the effect of the ortho substituent is general with respect to the aryl halide. Thus, a further enhancement of Z-selectivity (up to Z/E 100/0) was also observed with other ortho substituted aryl halides, regardless the electronic nature, and the size of the ortho substituent (e.g.; $\left.\mathrm{OBn}, \mathrm{Me}, \mathrm{NO}_{2}, i \mathrm{Pr}\right)$. 
Table 1. One-pot three-steps tandem synthesis of $Z$-trisubstituted olefins 2

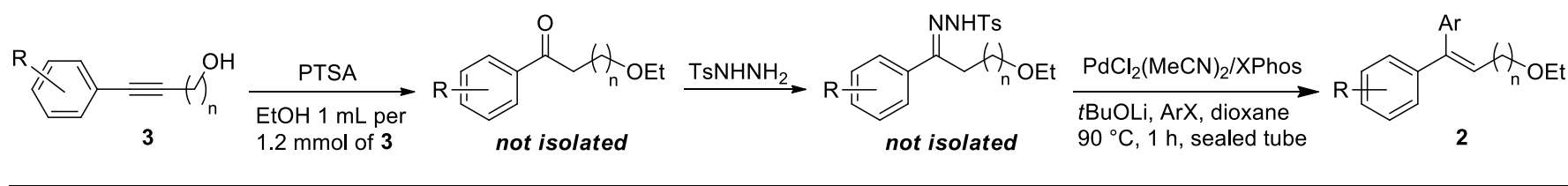

\begin{tabular}{lll}
\hline Entry & Alkyne 3 & Aryl halide \\
\hline &
\end{tabular}

3a

2

3<smiles>COc1ccc2ccc(Br)cc2c1</smiles>

4

5

6

7

8

10<smiles>COc1ccc(C#CCCO)cc1</smiles>

3b<smiles>COc1cccc(I)c1</smiles><smiles>Oc1ccccc1I</smiles><smiles>CCOCC=C(c1ccc(OC)cc1)c1cccc(OC)c1</smiles>

2b<smiles>CCOC/C=C(\c1ccc(OC)cc1)c1ccc2cc(OC)ccc2c1</smiles>

97

$55 / 45$<smiles>CCOC/C=C(\c1ccc(OC)cc1)c1ccccc1OC</smiles>

2d<smiles>CCOC/C=C(/c1ccc(OC)cc1)c1ccccc1OCC</smiles>

$97 / 3$<smiles>CCOC/C=C(\c1ccc(OC)cc1)c1ccc(OC)cc1[N+](=O)[O-]</smiles>

$2 f$<smiles>CCO/C=C(/c1ccc(OC)cc1)c1cccc(C)c1C</smiles><smiles>CCOC/C=C(/c1ccc(OC)cc1N)c1c(C)cc([N+](=O)[O-])cc1OC</smiles>

$2 \mathrm{~h}$

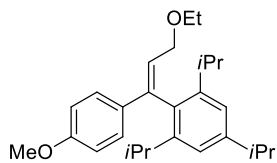

$2 \mathrm{i}$<smiles>CCOCC/C=C(/c1ccc(OC)cc1)c1c(OC)ccc2ccccc12</smiles>

58

$98 / 2$ 
11 $"$

12

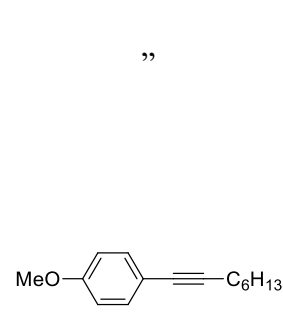

$3 c$

14

15

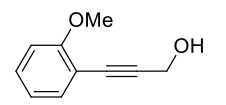

3d<smiles>CCOCC/C=C(/c1ccc(OC)cc1)c1ccccc1C</smiles><smiles>COc1ccc(I)c([N+](=O)[O-])c1</smiles><smiles>COc1ccc(I)c(OC)c1</smiles><smiles>COc1ccc(I)c([N+](=O)[O-])c1</smiles><smiles>COc1cccc(I)c1</smiles>

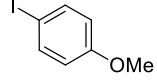

2k<smiles>C/C=C(\c1ccc(OC)cc1)c1ccc(OC)cc1OC</smiles>

$2 m$<smiles></smiles>

2n<smiles>CCOC/C=C(\c1cccc(OC)c1)c1ccccc1OC</smiles>

20<smiles>CCOC/C=C(/c1ccc(OC)cc1)c1ccccc1OC</smiles>

Scheme 1. Proposed mechanism for the synthesis of $\mathbf{2 d}$ from 3a or 3d.

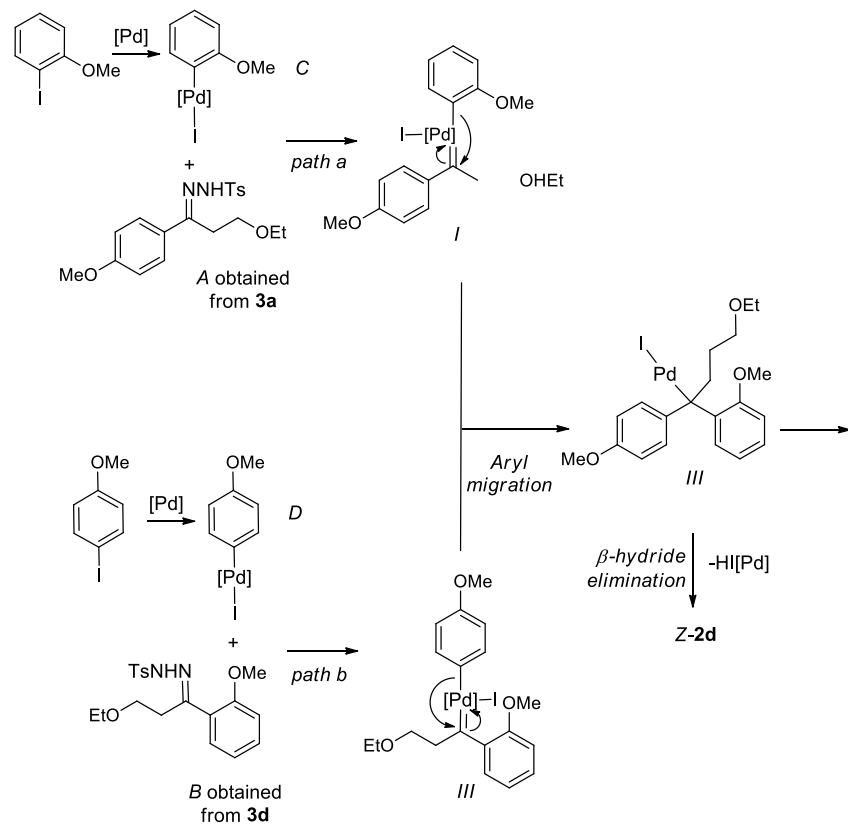

involves a same alkylpalladium intermediate III (Scheme 1). In the coupling step between the ortho arylpalladium species $C$ and the para $N$-tosylhydrazone $A$ obtained from 3a, a Pd carbene complex of type $I$ would be formed (path $a$ ). This intermediate may evolved into an alkylpalladium complex III through a migratory of the 
ortho anisole nucleus. According to this mechanism, the same alkylpalladium complex $I I I$ is formed from the coupling of the ortho substituted- $N$-tosylhydrazone $B$ with the para substituted palladium species $D$ through the Pd-carbene species II (path $b$ ). A further $\beta$-hydride elimination on III would give olefin $\mathbf{2 d}$ with the same Z-selectivity together with the regeneration of the $\operatorname{Pd}(0)$ catalyst in the presence of $t \mathrm{BuOLi}$.

We next focused our attention on the preparation of olefins $\mathbf{2 p}$ $\mathbf{z}$ having an ortho-OMOM substituent, which could be further transformed into 4-aryl-2 $H$-chromenes 1a-k by an intramolecular cyclization. As depicted in Table 2, using the optimized conditions, olefins 2p-z were obtained again with a pronounced $Z$ stereoselectivities ranging from $Z / E$ : $90 / 10$ to $99 / 1$. One can note that this one-pot three-steps process worked well with various substituted aryl halides and resulted with no exception in the formation of the expected Z-olefins $\mathbf{2 p - z}$ in good to excellent overall yields (50\% to $94 \%$ ) indicating that several groups (e.g.;
$\mathrm{Me}, \mathrm{OMe}, \mathrm{OMOM}, \mathrm{Cl}, \mathrm{F}, \mathrm{CN}$ ) are well tolerated. Having ortho OMOM diarylethylenes $\mathbf{2 p - z}$ in hands, the cyclization reaction was attempted in refluxing EtOH in the presence of PTSA (3.0 equiv.). As expected, 4-aryl- $2 H$-chromenes 1a-k were obtained in good to excellent yields within a short reaction time of $1 \mathrm{~h}$. Thus, a small library of 4-aryl-2H-chromenes was prepared rapidly from arylalkynols 3 with efficiency and a reduced number of purification steps. This success prompted us to extend the versatility of the method to the preparation of 5-aryl-2,3-dihydrobenzo[ $b]$ oxepine 4 (Scheme 2). Thus, starting from arylalkynol 3b having a butynol chain, and using 1-iodo-2-methoxymethoxybenzene as coupling partner, the one-pot process led to Z-olefin $\mathbf{5}$ in a good $72 \%$ yield and high stereoselectivity (Z/E: 95/5). In the case of olefin $\mathbf{5}$, TfOH was found to be superior to PTSA to achieve the cyclization reaction, providing the expected benzoxepine 4 in an acceptable $62 \%$ yield.

Table 2. Synthesis of 4-aryl-2H-chromenes 1a-k

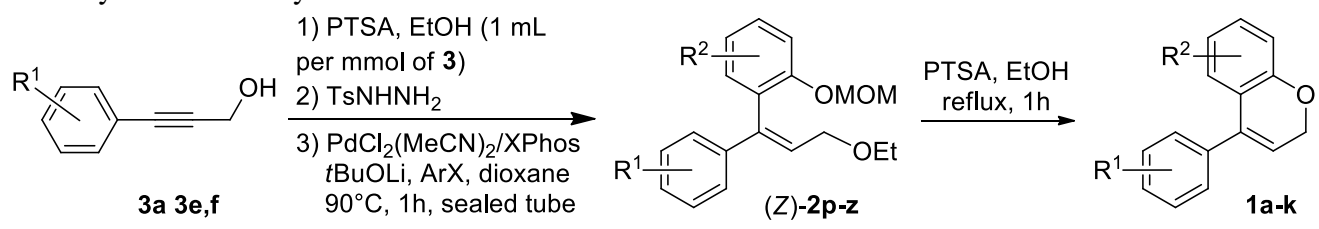

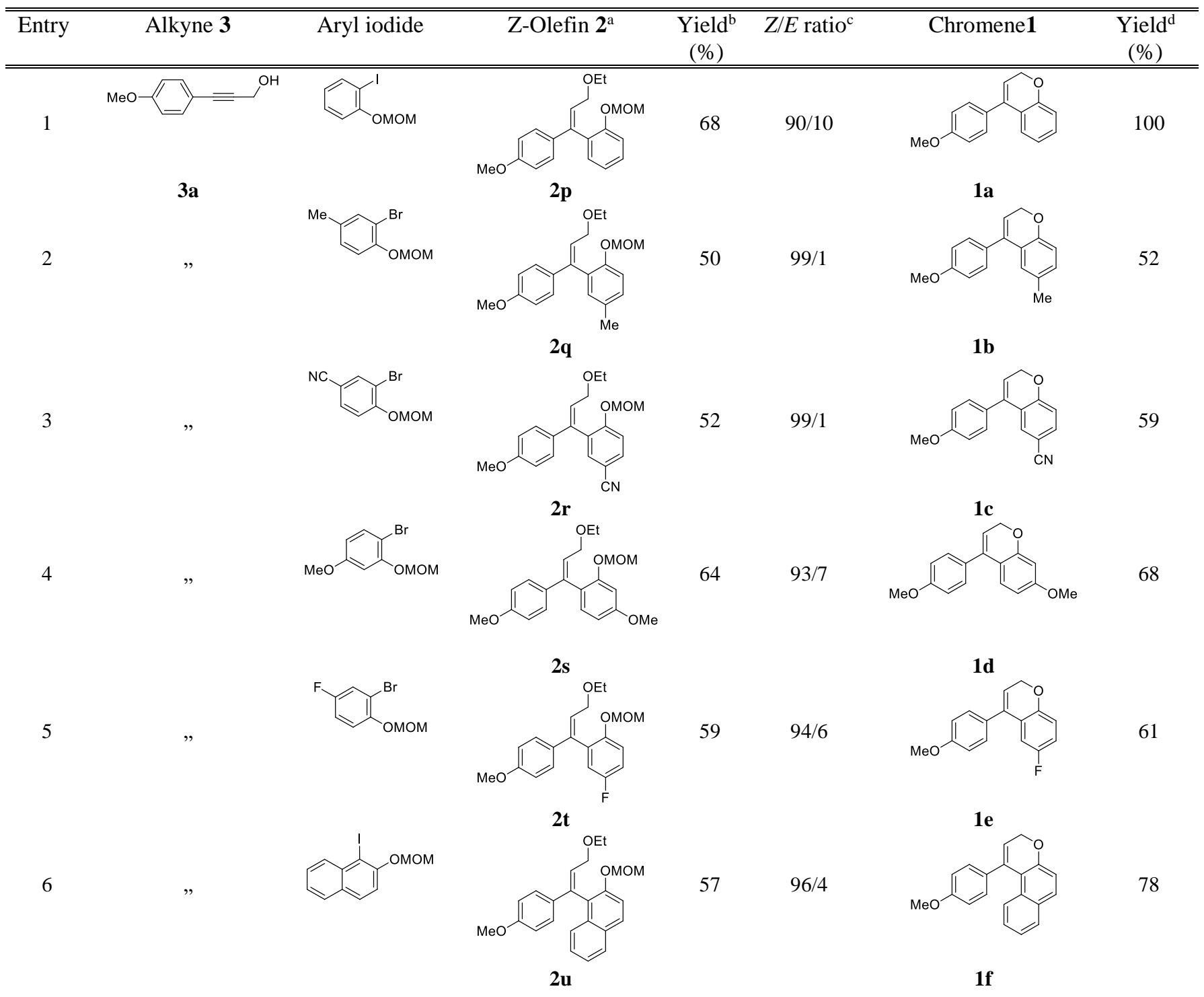


7<smiles>COc1cc(C#CCO)cc(OC)c1OC</smiles><smiles>COc1ccc(C#N)cc1Br</smiles>

$3 e$

8<smiles>COc1ccc2ccccc2c1I</smiles>

9<smiles>COc1ccc(I)c(OC)c1Br</smiles><smiles>CCOC/C=C(/c1cc(OC)c(OC)c(OC)c1)c1ccc(OC)c(Br)c1OC</smiles><smiles>Cc1ccc(C#CCO)cc1</smiles>

10<smiles>COc1ccc2ccccc2c1I</smiles>

3f 2x<smiles>CCOCC=C(c1cc(OC)c(OC)c(OC)c1)c1cc(C#N)ccc1OC</smiles>

2v<smiles>CCOCC=C(c1cc(OC)c(OC)c(OC)c1)c1c(OC)ccc2ccccc12</smiles>

$2 w$<smiles>CCOC/C=C(/c1ccc(C)cc1)c1c(OC)ccc2ccccc12</smiles>

$2 y$<smiles>CCOC/C=C(/c1ccc(C)cc1)c1ccc(OC)cc1OC</smiles>

80

93/7

$98 / 2$<smiles>COc1cc(C2=CCOc3ccc(C#N)cc32)cc(OC)c1OC</smiles>

$1 \mathrm{~g}$<smiles>COc1cc(C2=CCOc3ccc4ccccc4c32)cc(OC)c1OC</smiles>

11<smiles>COc1ccc(I)c(OC)c1</smiles>

$2 z$

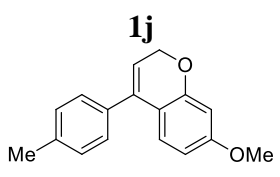

$1 \mathrm{k}$

aThe stereochemistry of the double bond was determined in all cases by NOESY experiments.

${ }^{b}$ Overall isolated yield based from arylalkynols $\mathbf{3 a}, \mathbf{3 e}$ and $\mathbf{3 f}$ for the three step-sequence.

${ }^{\mathrm{c}} Z / E$ ratio was determined by ${ }^{1} \mathrm{H} \mathrm{NMR}$ in the crude reaction mixture.

${ }^{\mathrm{d}}$ Isolated yields of $\mathbf{1}$.

Scheme 2. Synthesis of $\mathbf{4}$ from alkynol $\mathbf{3 b}$.
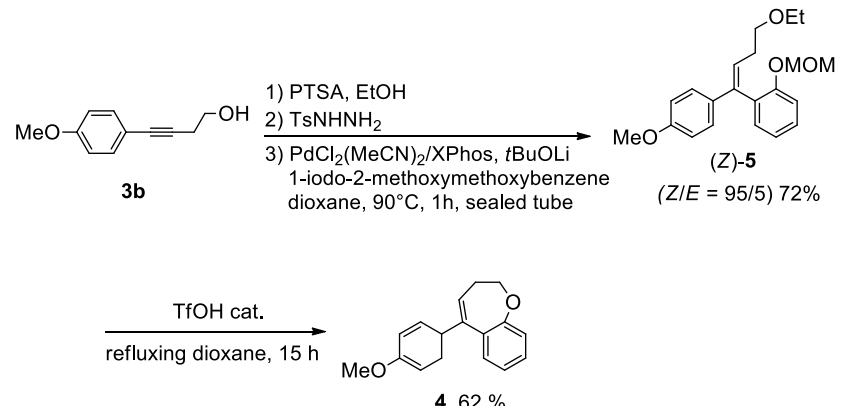

\section{Conclusion}

In summary, we have described a rapid and stereoselective access to $Z$-trisubstituted olefins $\mathbf{2}$ from arylalkynes using a onepot three steps procedure based on alkyne hydration, $\mathrm{N}$ tosylhydrazone in situ formation and subsequent Pd-coupling reactions with aryl halides. A remarkable to exclusive $Z$-selectivity in olefins 2 formation was observed with aryl halides as well as with arylalkynes having an ortho substituent. This protocol proved to be suitable for the synthesis of a series of olefins $\mathbf{2} \mathbf{p}-\mathbf{z}$, having an ortho-OMOM substituent, which were next transformed into the corresponding 4-aryl-2H-chromenes 1a-k in good to excellent yields using PTSA in refluxing EtOH.
Owing to its practical advantages and low environmental impact, the exploitation of this new procedure, in the context of our current medicinal program, will allow us to quickly prepare a small library of novel olefins of type $\mathbf{2}$, as well as 4 -aryl- $2 \mathrm{H}$-chromenes $\mathbf{1}$ bearing adequate decorations. These compounds which could be regarded as isoCA-4 and restricted-isoCA-4 analogues, respectively, are under investigation in our laboratory.

\section{Experimental Section}

\section{General comments}

All glasswares were oven-dried at $140{ }^{\circ} \mathrm{C}$ and all reactions were conducted under an argon atmosphere. Solvents: cyclohexane and ethyl acetate (EtOAc), for chromatography were technical grade. All new compounds were characterized by ${ }^{1} \mathrm{H}-\mathrm{NMR},{ }^{13} \mathrm{C}-\mathrm{NMR}$, and IR spectroscopy. ${ }^{1} \mathrm{H}$ and ${ }^{13} \mathrm{C}$ NMR spectra were measured in $\mathrm{CDCl}_{3}$ with a Bruker Avance 300. ${ }^{1} \mathrm{H}$ chemical shifts are reported in ppm from an internal standard TMS or of residual chloroform (7.27 ppm). The following abreviation are used: $\mathrm{m}$ (multiplet), s (singlet), d (doublet), t (triplet), $\mathrm{q}$ (quadruplet), dd (doublet of doublet), dt (doublet of triplet). ${ }^{13} \mathrm{C}$ chemical shifts are reported in ppm from the central peak of deuteriochloroform (77.14). IR spectra were measured on a Bruker Vector 22 spectrophotometer (neat, $\mathrm{cm}^{-1}$ ). Analytical TLC was performed on Merck precoated silica gel 60 F-254 plates. Merck silica gel 60 (230-400 mesh) was used for column chromatography. The plates were visualized by either UV light $(254 \mathrm{~nm})$, or by a solution of phosphomolybdic acid in ethanol. Melting points were recorded on a Büchi B-450 apparatus and are uncorrected.

Typical procedure for Sonogashira-Linstrumelle couplings: A mixture of 4-iodoanisole (42.8 mmol, 1 equiv.), $\mathrm{PdCl}_{2}\left(\mathrm{PPh}_{3}\right)_{2}$ (2.14 mmol, 0.05 equiv.) and $\mathrm{CuI}(3.42 \mathrm{mmol}, 0.08$ equiv.) under argon atmosphere was 
dissolved in anhydrous triethylamine $(100 \mathrm{~mL})$. Suitable alkyne (51.4 mmol, 1.2 equiv.) was then added dropwise. After being stirred at room temperature for $4 \mathrm{~h}$, the solution was quenched with a saturated $\mathrm{NH}_{4} \mathrm{Cl}$ solution and extracted with $\mathrm{Et}_{2} \mathrm{O}(3 \times 50 \mathrm{~mL})$. Organic layers were then washed with brine, dried over $\mathrm{Na}_{2} \mathrm{SO}_{4}$ and evaporated in vacuo to give the crude product which was purified by flash chromatography.

\section{3-(4-Methoxyphenyl)prop-2-yn-1-ol (3a)}

Yield: $99 \%$; yellow solid; $\mathrm{F}=66^{\circ}-69^{\circ} \mathrm{C}\left(62.5^{\circ}-64.5^{\circ} \mathrm{C}\right)^{[16]} ; \mathrm{R}_{\mathrm{f}}: 0.5$ (EtOAc/c-hexane: 4/6); IR ( $\left.v, \mathrm{~cm}^{-1}\right) 3261,2232,1603,1506,1248,1015$, $835 ; \delta_{\mathrm{H}}\left(300 \mathrm{MHz}, \mathrm{CDC1}_{3}\right) 7.37(\mathrm{~d}, 2 \mathrm{H}, J=8.8 \mathrm{~Hz}), 6.84(\mathrm{~d}, 2 \mathrm{H}, J=8.8$ $\mathrm{Hz}), 4.48(\mathrm{~s}, 2 \mathrm{H}), 3.81(\mathrm{~s}, 3 \mathrm{H}), \mathrm{OH}$ not seen; $\delta_{\mathrm{C}}\left(75 \mathrm{MHz}, \mathrm{CDCl}_{3}\right) 159.7$, 133.1 (2C), 114.6, 113.9 (2C), 85.8, 85.6, 55.2, 51.7; MS (APCI+) $\mathrm{m} / \mathrm{z}$ $163.0\left(\mathrm{MH}^{+}\right)$. The spectral data above are in accordance with the literature data. ${ }^{[17]}$

\section{4-(4-Methoxyphenyl)but-3-yn-1-ol (3b)}

Yield: $89 \%$; yellow solid; $\mathrm{F}=60^{\circ}-61^{\circ} \mathrm{C}\left(61^{\circ} \mathrm{C}\right)^{[18]} ; \mathrm{R}_{\mathrm{f}}$ : $0.5(\mathrm{EtOAc} / \mathrm{c}$ hexane: 4/6); IR $\left(v, \mathrm{~cm}^{-1}\right) 2836,1674,1602,1573,1509,1463,1440,1242$, 1029,$830 ; \delta_{\mathrm{H}}\left(300 \mathrm{MHz}, \mathrm{CDC1}_{3}\right) 7.35(\mathrm{~d}, 2 \mathrm{H}, J=8.8 \mathrm{~Hz}), 6.82(\mathrm{~d}, 2 \mathrm{H}, J=$ $8.8 \mathrm{~Hz}), 3.80(\mathrm{~m}, 5 \mathrm{H}), 2.67(\mathrm{t}, 2 \mathrm{H}, J=6.2 \mathrm{~Hz}), \mathrm{OH}$ not seen; $\delta_{\mathrm{C}}(75 \mathrm{MHz}$ $\left.\mathrm{CDCl}_{3}\right)$ 159.3, 132.9 (2C), 115.4, 113.8 (2C), 84.7, 82.2, 61.2, 55.2, 23.8; MS (APCI+) $m / z 177.2\left(\mathrm{MH}^{+}\right)$. The spectral data above are in accordance with the literature data. ${ }^{[19]}$

\section{1-Methoxy-4-(oct-1-yn-1-yl)benzene (3c)}

Yield: 100\%; brown oil; $\mathrm{R}_{\mathrm{f}}$ : 0.5 (EtOAc/c-hexane: 5/95); IR $\left(\mathrm{v}, \mathrm{cm}^{-1}\right)$ 2934, 2197, 1509, 1246, 904, 831, 729, 620; $\delta_{\mathrm{H}}\left(300 \mathrm{MHz}, \mathrm{CDC1}_{3}\right) 7.32(\mathrm{~d}$, $2 \mathrm{H}, J=8.0 \mathrm{~Hz}), 6.81(\mathrm{~d}, 2 \mathrm{H}, J=8.0 \mathrm{~Hz}), 3.79(\mathrm{~s}, 3 \mathrm{H}), 2.38(\mathrm{t}, 2 \mathrm{H}, J=6.9$ $\mathrm{Hz}), 1.60-1.31(\mathrm{~m}, 8 \mathrm{H}), 0.90(\mathrm{t}, 3 \mathrm{H}, J=6.3 \mathrm{~Hz}) ; \delta_{\mathrm{C}}\left(75 \mathrm{MHz}, \mathrm{CDCl}_{3}\right)$ $159.1,133.0$ (2C), 116.4, 113.9 (2C), 88.9, 80.3, 55.3, 31.5, 29.0, 28.8, $22.7,19.5,14.2 ; \mathrm{MS}(\mathrm{APCI}+) \mathrm{m} / z 217.5\left(\mathrm{MH}^{+}\right)$. The spectral data above are in accordance with the literature data. ${ }^{[20]}$

\section{3-(2-Methoxyphenyl)prop-2-yn-1-ol (3d)}

Yield: 87\%; light yellow oil; $\mathrm{R}_{\mathrm{f}}$ : 0.3 (EtOAc/c-hexane: 3/7); IR ( $v$ $\left.\mathrm{cm}^{-1}\right) 2136,1509,1467,1246,904,825,755,729 ; \delta_{\mathrm{H}}\left(300 \mathrm{MHz}, \mathrm{CDC}_{3}\right)$ $7.35(\mathrm{dd}, 1 \mathrm{H}, J=7.2,2.9 \mathrm{~Hz}), 7.22(\mathrm{~m}, 1 \mathrm{H}), 6.85-6.70(\mathrm{~m}, 2 \mathrm{H}), 4.48(\mathrm{~d}$, $2 \mathrm{H}, J=6.8 \mathrm{~Hz}), 3.8(\mathrm{~s}, 3 \mathrm{H}), 2.23(\mathrm{t}, 1 \mathrm{H}, J=6.8 \mathrm{~Hz}) ; \delta_{\mathrm{C}}\left(75 \mathrm{MHz}, \mathrm{CDCl}_{3}\right)$ 159.9, 133.7, 129.9, 120.5, 111.7, 110.6, 91.5, 81.8, 55.8, 51.7; MS (ESI+) $\mathrm{m} / \mathrm{z} 163.2\left(\mathrm{MH}^{+}\right)$. The spectral data above are in accordance with the literature data. ${ }^{[21]}$

\section{General procedure for the synthesis of trisubstituted olefins:}

To an Emrys Optimizer 0.5-2 $\mathrm{mL}$ pyrex reaction vessel were added alkyne (1.2 mmol, 1 equiv.) and PTSA. $\mathrm{H}_{2} \mathrm{O}(0.24 \mathrm{mmol}, 0.20$ equiv.) in EtOH $(1 \mathrm{~mL})$. The reaction vessel was then placed in the Emrys Optimizer and exposed to microwave irradiation according to the following specifications: temperature, $120{ }^{\circ} \mathrm{C}$; time, $30 \mathrm{~min}$; fixed hold time, on; sample absorption, high; pre-stirring, $60 \mathrm{~s}$. After cooling to room temperature, suitable sulfonhydrazide ( $1.35 \mathrm{mmol}, 1.1$ equiv.) was added and the reaction vessel was exposed once again to microwave irradiation according to the following specifications: temperature, $50{ }^{\circ} \mathrm{C}$; time, $15 \mathrm{~min}$; fixed hold time, on; sample absorption, high; pre-stirring, $60 \mathrm{~s}$. After cooling to room temperature, $\mathrm{PdCl}_{2}\left(\mathrm{MeCN}_{2}\right.$ ( $0.12 \mathrm{mmol}, 0.10$ equiv.), $\mathrm{Xphos}(0.24 \mathrm{mmol}, 0.20$ equiv.), $t \mathrm{BuOLi}(6 \mathrm{mmol}, 5$ equiv.) and dioxane (1 $\mathrm{mL}$ ) were added, and the mixture was stirred at room temperature. After 3 min, the aryl halide ( $1.5 \mathrm{mmol}, 1.25$ equiv.) was finally added, the vessel was sealed and the mixture was stirred at $90{ }^{\circ} \mathrm{C}$ for $1 \mathrm{~h}$. After cooling to room temperature, $\mathrm{H}_{2} \mathrm{O}$ was added and the mixture was extracted with $\mathrm{CH}_{2} \mathrm{Cl}_{2}(3 \times 2 \mathrm{~mL})$. Organic layers were dried, concentrated, and the crude product was purified by column chromatography on silica gel. All the compounds gave satisfactory spectroscopic data.

\section{4,4'-(3-Ethoxyprop-1-ene-1,1-diyl)bis(methoxybenzene) (2a)}

Yield: 63\%; colorless oil; $\mathrm{R}_{\mathrm{f}}: 0.67$ (EtOAc/c-hexane: 1/9); IR ( $\left.v, \mathrm{~cm}^{-1}\right)$ 2972, 2836, 2228, 2066, 2033, 2011, 1606, 1575, 1510, 1463, 1418, 1374 $1286,1244,1174,1098,1034,835,779,737 ; \delta_{\mathrm{H}}\left(300 \mathrm{MHz}, \mathrm{CDC1}_{3}\right) 7.20$ $(\mathrm{d}, 2 \mathrm{H}, J=8.8 \mathrm{~Hz}), 7.10(\mathrm{~d}, 2 \mathrm{H}, J=8.7 \mathrm{~Hz}), 6.90(\mathrm{~d}, 2 \mathrm{H}, J=8.7 \mathrm{~Hz}), 6.81$ $(\mathrm{d}, 2 \mathrm{H}, J=8.8 \mathrm{~Hz}), 6.09(\mathrm{t}, 1 \mathrm{H}, J=6.7 \mathrm{~Hz}), 4.03(\mathrm{~d}, 2 \mathrm{H}, J=6.7 \mathrm{~Hz}), 3.84$ $(\mathrm{s}, 3 \mathrm{H}), 3.80(\mathrm{~s}, 3 \mathrm{H}), 3.47(\mathrm{q}, 2 \mathrm{H}, J=7.0 \mathrm{~Hz}), 1.20(\mathrm{t}, 3 \mathrm{H}, J=7.0 \mathrm{~Hz}) ; \delta_{\mathrm{C}}$ $\left(75 \mathrm{MHz}, \mathrm{CDCl}_{3}\right) 159.1,158.9,143.7,134.9,131.8(2 \mathrm{C}), 130.9(2 \mathrm{C})$, 128.8, 123.7, 113.4 (4C), 68.4, 65.6, 55.2 (2C), 15.3; MS (APCI+) $\mathrm{m} / \mathrm{z}$ 299.6 $\left(\mathrm{MH}^{+}\right)$. Anal. Calcd for $\mathrm{C}_{19} \mathrm{H}_{22} \mathrm{O}_{3}$ : C, 76.48; H, 7.43, found: C, 76.41; $\mathrm{H}, 7.38$. (2b)

Yield: 78\%; colorless oil; $\mathrm{R}_{\mathrm{f}}$ : 0.33 (EtOAc/c-hexane: 4/6); IR $\left(v, \mathrm{~cm}^{-1}\right)$ 2972, 2836, 2228, 2066, 2033, 1510, 1463, 1418, 1374, 1286, 1244, 1174, $936,835,779,737 ; \delta_{\mathrm{H}}\left(300 \mathrm{MHz}, \mathrm{CDC1}_{3}\right)$ [the presence of $Z / E$ isomers in a ratio of $65 / 35$ complicates the spectrum] major $Z$ isomer $7.93-7.12(\mathrm{~m}$, $3 \mathrm{H}), 7.76-7.00(\mathrm{~m}, 5 \mathrm{H}), 6.29-6.15(\mathrm{~m}, 1 \mathrm{H}), 4.01(\mathrm{~d}, 2 \mathrm{H}, J=6.7 \mathrm{~Hz})$, $3.90(\mathrm{~s}, 6 \mathrm{H}), 3.53(\mathrm{q}, 2 \mathrm{H}, J=7.0 \mathrm{~Hz}), 1.23(\mathrm{t}, 3 \mathrm{H}, J=7.0 \mathrm{~Hz})$; minor $E$ isomer [only the most significant signals are listed] $6.29-6.15(\mathrm{~m}, 1 \mathrm{H})$, $4.06(\mathrm{~d}, 2 \mathrm{H}, J=6.7 \mathrm{~Hz}), 3.84(\mathrm{~s}, 3 \mathrm{H}), 3.76(\mathrm{~s}, 3 \mathrm{H}), 3.50-3.43(\mathrm{~m}, 2 \mathrm{H})$, $1.24-1.18(\mathrm{~m}, 3 \mathrm{H}) ; \delta_{\mathrm{C}}\left(75 \mathrm{MHz}, \mathrm{CDCl}_{3}\right)$; major $Z$ isomer $159.5,159.3$, $143.9,141.1,134.4,129.2,128.7$ (2C), 124.1, 122.4, 115.3, 113.6 (2C), $113.1,68.5,65.8,55.4$ (2C), 15.4; minor $E$ isomer [only the most significant signals are listed] $159.09,144.0,143.9,131.6,131.1$ (2C), $125.8,120.5,113.5,113.0,68.5,65.87,55.4(2 \mathrm{C}), 15.44$; MS (APCI+) $\mathrm{m} / \mathrm{z}$ $299.8\left(\mathrm{MH}^{+}\right)$. Anal. Calcd for $\mathrm{C}_{19} \mathrm{H}_{22} \mathrm{O}_{3}: \mathrm{C}, 76.48 ; \mathrm{H}, 7.43$, found: C, 76.24; $\mathrm{H}, 7.21$.

(Z)-2-(3-Ethoxy-1-(4-methoxyphenyl)prop-1-en-1-yl)-6-methoxy naphthalene (2c)

Yield: 97\%; yellow oil; $\mathrm{R}_{\mathrm{f}}$ : 0.6 (EtOAc/c-hexane: 2/8); IR $\left(v, \mathrm{~cm}^{-1}\right)$ 2931, 2339, 2136, 1509, 1467, 1410, 1334, 1238, 1197, 1153, 1128, 1104, $1047,1013,924,750,646 ; \delta_{\mathrm{H}}\left(300 \mathrm{MHz}, \mathrm{CDC1}_{3}\right)$ [the presence of $Z / E$ isomers in a ratio of 55/45 complicates the spectrum] Major $Z$ isomer 7.74 $-7.59(\mathrm{~m}, 3 \mathrm{H}), 7.48(\mathrm{dd}, 1 \mathrm{H}, J=8.6,1.8 \mathrm{~Hz}), 7.30-7.10(\mathrm{~m}, 4 \mathrm{H}), 6.95(\mathrm{q}$, $2 \mathrm{H}, J=8.8 \mathrm{~Hz}), 6.31(\mathrm{t}, 1 \mathrm{H}, J=6.8 \mathrm{~Hz}), 4.13(\mathrm{~d}, 2 \mathrm{H}, J=6.8 \mathrm{~Hz}), 3.92(\mathrm{~s}$, $3 \mathrm{H}), 3.88(\mathrm{~s}, 3 \mathrm{H}), 3.53(\mathrm{t}, 2 \mathrm{H}, J=7.0 \mathrm{~Hz}), 1.25(\mathrm{t}, 3 \mathrm{H}, J=7.0 \mathrm{~Hz})$; minor $E$ isomer [only the most significant signals are listed] $6.24(\mathrm{t}, 1 \mathrm{H}, J=6.8 \mathrm{~Hz})$, $4.09(\mathrm{~d}, 2 \mathrm{H}, J 6.8 \mathrm{~Hz}), 3.96(\mathrm{~s}, 3 \mathrm{H}), 3.81(\mathrm{~s}, 3 \mathrm{H}), 3.48(\mathrm{q}, 2 \mathrm{H}, J=7.0 \mathrm{~Hz})$, $1.22(\mathrm{t}, 3 \mathrm{H}, J=7.0 \mathrm{~Hz}) ; \delta_{\mathrm{C}}\left(75 \mathrm{MHz}, \mathrm{CDCl}_{3}\right)$ major $E$ isomer $159.1,157.9$, $144.2,137.7,134.1,133.9,131.8,131.2$ (2C), 128.8, 128.7, 126.9, 126.3, $125.3,118.9,113.6$ (2C), 105.7, 68.6, 65.8, 55.5, 55.4, 15.4; minor $Z$ isomer $159.3,158.0,144.2,126.3,134.8,134.8,134.1,133.9,129.0(2 \mathrm{C})$, $128.8,128.7,124.3,119.1,113.6(2 \mathrm{C}), 105.8,68.6,65.8,55.5,55.4,55.4$, 15.4. MS (APCI+) $m / z, 349.0\left(\mathrm{MH}^{+}\right)$. Anal. Calcd for $\mathrm{C}_{23} \mathrm{H}_{24} \mathrm{O}_{3}: \mathrm{C}, 79.28 ; \mathrm{H}$, 6.94, found: C, 79.12; H, 6.81 (2d)

(Z)-1-(3-Ethoxy-1-(4-methoxyphenyl)prop-1-en-1-yl)-2-methoxybenzene

Yield: 77\%; brownish oil; $\mathrm{R}_{\mathrm{f}}$ : 0.45 (EtOAc/c-hexane: 2/8); IR ( $\left.v, \mathrm{~cm}^{-1}\right)$ 2836, 2228, 2033, 2011, 1606, 1510, 1463, 1418, 1415, 1374, 1286, 1174, $1098,1034,779,735 ; \delta_{\mathrm{H}}\left(300 \mathrm{MHz}, \mathrm{CDC1}_{3}\right)$ only the major $Z$ isomer is described $7.33(\mathrm{~m}, 1 \mathrm{H}), 7.19(\mathrm{~d}, 2 \mathrm{H}, J=8.9 \mathrm{~Hz}), 7.09(\mathrm{dd}, 1 \mathrm{H}, J=7.4,1.8$ $\mathrm{Hz}), 7.02-6.85(\mathrm{~m}, 2 \mathrm{H}), 6.79(\mathrm{~d}, 2 \mathrm{H}, J=8.9 \mathrm{~Hz}), 6.25(\mathrm{t}, 1 \mathrm{H}, J=6.5 \mathrm{~Hz})$, $3.91(\mathrm{~d}, 2 \mathrm{H}, J=6.5 \mathrm{~Hz}), 3.78(\mathrm{~s}, 3 \mathrm{H}), 3.70(\mathrm{~s}, 3 \mathrm{H}), 3.42(\mathrm{q}, 2 \mathrm{H}, J=7.0 \mathrm{~Hz})$, $1.17(\mathrm{t}, 3 \mathrm{H}, J=7.0 \mathrm{~Hz})$; $\delta_{\mathrm{C}}\left(75 \mathrm{MHz}, \mathrm{CDCl}_{3}\right)$ only the major $Z$ isomer is described 158.9, 157.0, 139.4, 133.7, 131.2, 128.9, 128.2, 127.1 (2C), $124.1,120.5,113.4(2 \mathrm{C}), 111.1,68.6,65.4,55.6,55.2,15.3 ; \mathrm{MS}(\mathrm{ESI}+) \mathrm{m} / \mathrm{z}$ $321.1\left(\mathrm{MNa}^{+}\right)$. Anal. Calcd for $\mathrm{C}_{19} \mathrm{H}_{22} \mathrm{O}_{3}: \mathrm{C}, 76.48 ; \mathrm{H}, 7.43$, found: $\mathrm{C}$, $76.33 ; \mathrm{H}, 7.27$.

(Z)-1-(benzyloxy)-2-(3-ethoxy-1-(4-methoxyphenyl)prop-1-en-1-yl) benzene $(\mathbf{2 e})$

Yield: 51\%; brownish oil; $\mathrm{R}_{\mathrm{f}}$ : 0.45 (EtOAc/c-hexane: $\left.2 / 8\right)$; IR $\left(v, \mathrm{~cm}^{-1}\right)$ $2053,1606,1374,1286,1244,1098,1072,1036,837,803,749,648 ; \delta_{\mathrm{H}}$ $\left(300 \mathrm{MHz}, \mathrm{CDCl}_{3}\right)$ Only the major $Z$ isomer is described $7.39-7.17(\mathrm{~m}$, $7 \mathrm{H}), 7.13-7.08(\mathrm{~m}, 2 \mathrm{H}), 7.03(\mathrm{~m}, 2 \mathrm{H}), 6.84(\mathrm{~d}, 2 \mathrm{H}, J=8.6 \mathrm{~Hz}), 6.26(\mathrm{t}$, $1 \mathrm{H}, J=6.5 \mathrm{~Hz}), 4.99(\mathrm{~s}, 2 \mathrm{H}), 3.98(\mathrm{~d}, 2 \mathrm{H}, J=6.5 \mathrm{~Hz}), 3.83(\mathrm{~s}, 3 \mathrm{H}), 3.45(\mathrm{q}$, $2 \mathrm{H}, J=7.0 \mathrm{~Hz}), 1.18(\mathrm{t}, 3 \mathrm{H}, J=7.0 \mathrm{~Hz}) ; \delta_{\mathrm{C}}\left(75 \mathrm{MHz}, \mathrm{CDCl}_{3}\right)$ only the major $Z$ isomer is described 159.1, 156.2, 140.2, 137.2, 134.3, 131.6, 129.0, 128.8, 128.4 (2C), 127.9 (2C), 127.6, 127.0 (2C), 124.9, 120.8, 113.6 (2C), 112.9, 70.1, 68.8, 65.6, 55.4, 15.4; MS (APCI+) $m / z 375.4\left(\mathrm{MH}^{+}\right)$. Anal. Calcd for $\mathrm{C}_{25} \mathrm{H}_{26} \mathrm{O}_{3}: \mathrm{C}, 80.18 ; \mathrm{H}, 7.00$, found: C, 79.67; H, 6.79.

(Z)-1-(3-Ethoxy-1-(4-methoxyphenyl)prop-1-enyl)-4-methoxy-2-nitro benzene $(\mathbf{2 f})$

Yield: 97\%; brownish oil; $\mathrm{R}_{\mathrm{f}}$ : 0.63 (EtOAc/c-hexane: 2/8); IR $\left(\mathrm{v}, \mathrm{cm}^{-1}\right)$ $2972,1606,1529,1510,1462,1353,1246,1181,1099,1032,827,804 ; \delta_{\mathrm{H}}$ $\left(300 \mathrm{MHz}, \mathrm{CDC1}_{3}\right) 7.56(\mathrm{~d}, 1 \mathrm{H}, J=2.4 \mathrm{~Hz}), 7.31-7.21(\mathrm{~m}, 2 \mathrm{H}), 7.16(\mathrm{~d}$, $2 \mathrm{H}, J=8.9 \mathrm{~Hz}), 6.81(\mathrm{~d}, 2 \mathrm{H}, J=8.9 \mathrm{~Hz}), 6.25(\mathrm{t}, 1 \mathrm{H}, J=6.8 \mathrm{~Hz}), 3.93(\mathrm{~s}$, $3 \mathrm{H}), 3.88(\mathrm{~d}, 2 \mathrm{H}, J=6.8 \mathrm{~Hz}), 3.79(\mathrm{~s}, 3 \mathrm{H}), 3.43(\mathrm{q}, 2 \mathrm{H}, J=7.0 \mathrm{~Hz}), 1.18(\mathrm{t}$, $3 \mathrm{H}, J=7.0 \mathrm{~Hz}) ; \delta_{\mathrm{C}}\left(75 \mathrm{MHz}, \mathrm{CDCl}_{3}\right) 159.5,159.4,149.7,139.9,133.4$, 132.6, 127.9 (2C), 127.5, 124.9, 119.6, 113.8 (2C), 109.3, 68.1, 65.7, 55.4, 55.0, 15.3; MS (APCI-) $\mathrm{m} / z 342\left(\mathrm{M}-\mathrm{H}^{-}\right)$. Anal. Calcd for $\mathrm{C}_{19} \mathrm{H}_{21} \mathrm{NO}_{5}$ : C, 66.46; H, 6.16; N, 4.08, found: C, 66.11; H, 6.01; N, 3.97. 
(Z)-1-(3-Ethoxy-1-(4-methoxyphenyl)prop-1-en-1-yl)-2,3-dimethyl benzene $(\mathbf{2 g})$

Yield: $88 \%$; yellow oil; $\mathrm{R}_{\mathrm{f}}$ : 0.8 (EtOAc/c-hexane: $\left.1 / 9\right) ; \mathrm{IR}\left(v, \mathrm{~cm}^{-1}\right)$ 2930, 1606, 1510, 1460, 1291, 1248, 1177, 1099, 1037, 910, 826, 793, 730; $\delta_{\mathrm{H}}\left(300 \mathrm{MHz}, \mathrm{CDC1}_{3}\right)$ [the presence of $\mathrm{Z} / E$ isomers in a ratio of $95 / 5$ complicates the spectrum] major $Z$ isomer $7.17(\mathrm{~d}, 2 \mathrm{H}, J=8.9 \mathrm{~Hz}), 7.15-$ $7.07(\mathrm{~m}, 2 \mathrm{H}), 6.93(\mathrm{dd}, 1 \mathrm{H}, J=6.7,2.3 \mathrm{~Hz}), 6.79(\mathrm{~d}, 2 \mathrm{H}, J=8.9 \mathrm{~Hz}), 6.26$ $(\mathrm{t}, 1 \mathrm{H}, J=6.4 \mathrm{~Hz}), 3.91-3.81(\mathrm{~m}, 2 \mathrm{H}), 3.78(\mathrm{~s}, 3 \mathrm{H}), 3.41(\mathrm{q}, 2 \mathrm{H}, J=7.0$ $\mathrm{Hz}), 2.30(\mathrm{~s}, 3 \mathrm{H}), 2.00(\mathrm{~s}, 3 \mathrm{H}), 1.17(\mathrm{t}, 3 \mathrm{H}, J=7.0 \mathrm{~Hz})$; minor $E$ isomer [only the most significant signals are listed] $5.78(\mathrm{t}, 1 \mathrm{H}, J=6.4 \mathrm{~Hz}), 4.19$ $(\mathrm{d}, 1 \mathrm{H}, J=6.4,5.5 \mathrm{~Hz}), 3.53(\mathrm{q}, 2 \mathrm{H}, J=7.0 \mathrm{~Hz}) ; 2.24(\mathrm{~s}, 3 \mathrm{H}), 1.98(\mathrm{~s}, 3 \mathrm{H})$, $1.23(\mathrm{t}, 3 \mathrm{H}, J=7.0 \mathrm{~Hz}) ; \delta_{\mathrm{C}}\left(75 \mathrm{MHz}, \mathrm{CDCl}_{3}\right)$ major $Z$ isomer $159.2,142.8$, $138.7,137.0,134.8,133.3,129.1,127.6,127.7$ (2C), 125.3, 123.7, 113.6 (2C), 68.7, 65.6, 55.2, 20.4, 16.2, 15.2; MS (ESI+) $\mathrm{m} / z$ 319.1 $\left(\mathrm{MNa}^{+}\right)$. Anal. Calcd for $\mathrm{C}_{20} \mathrm{H}_{24} \mathrm{O}_{2}$ : C, 81.04; H, 8.16, found: C, 80.89; H, 8.05.

(Z)-2-(3-Ethoxy-1-(4-methoxyphenyl)prop-1-en-1-yl)-1,3,5-trimethyl benzene $(\mathbf{2 h})$

Yield: $71 \%$; colorless oil; $\mathrm{R}_{\mathrm{f}}$ : 0.5 (EtOAc/c-hexane: 4/6); IR $\left(v, \mathrm{~cm}^{-1}\right)$ 2962, 2219, 2186, 2053, 1606, 1510, 1461, 1249, 1180, 1099, 1037, 828, 652,$617 ; \delta_{\mathrm{H}}\left(300 \mathrm{MHz}, \mathrm{CDCl}_{3}\right)$ only the major $Z$ isomer is described 7.16 $(\mathrm{d}, 2 \mathrm{H}, J=8.6 \mathrm{~Hz}), 6.90(\mathrm{~m}, 2 \mathrm{H}), 6.78(\mathrm{~d}, 2 \mathrm{H}, J=8.6 \mathrm{~Hz}), 6.33(\mathrm{t}, 1 \mathrm{H}, J=$ $6.3 \mathrm{~Hz}), 3.77(\mathrm{~m}, 5 \mathrm{H}), 3.40(\mathrm{q}, 2 \mathrm{H}, J=7.0 \mathrm{~Hz}), 2.32(\mathrm{~s}, 3 \mathrm{H}), 2.05(\mathrm{~s}, 6 \mathrm{H})$, $1.16(\mathrm{t}, 3 \mathrm{H}, J=7.0 \mathrm{~Hz}) ; \delta_{\mathrm{C}}\left(75 \mathrm{MHz}, \mathrm{CDCl}_{3}\right)$ only the major $Z$ isomer is described 159.2, 140.3, 136.7, 136.1 (2C), 135.3, 132.1, 128.3 (2C), 127.2 (2C), 123.6, 113.9 (2C), 69.0, 66.1, 55.4, 21.2, 19.9 (2C), 15.4; MS (ESI+) $\mathrm{m} / \mathrm{z} 311.2\left(\mathrm{MH}^{+}\right)$. Anal. Calcd for $\mathrm{C}_{21} \mathrm{H}_{26} \mathrm{O}_{2}: \mathrm{C}, 81.25 ; \mathrm{H}, 8.44$, found: $\mathrm{C}$, $81.00 ; \mathrm{H}, 8.19$.

(Z)-2-(3-Ethoxy-1-(4-methoxyphenyl)prop-1-en-1-yl)-1,3,5-triisopropyl benzene $(2 \mathbf{i})$

Yield: 27\%; colorless oil; $\mathrm{R}_{\mathrm{f}}: 0.73$ (EtOAc/c-hexane: 1/9); IR $\left(v, \mathrm{~cm}^{-1}\right)$ 2925, 2187, 1607, 1510, 1249, 1180, 1100, 1036, 827; $\delta_{\mathrm{H}}(300 \mathrm{MHz}$, $\mathrm{CDC}_{3}$ ) [the presence of $Z / E$ isomers in a ratio of $94 / 6$ complicates the spectrum] major $Z$ isomer $7.20(\mathrm{~d}, 2 \mathrm{H}, J=9.0 \mathrm{~Hz}), 7.03(\mathrm{~s}, 2 \mathrm{H}), 6.78(\mathrm{~d}$ $2 \mathrm{H}, J=9.0 \mathrm{~Hz}), 6.45(\mathrm{t}, 1 \mathrm{H}, J=6.5 \mathrm{~Hz}), 3.82-3.72(\mathrm{~m}, 5 \mathrm{H}), 3.41(\mathrm{q}, 2 \mathrm{H}$, $J=7.0 \mathrm{~Hz}), 3.00-2.81(\mathrm{~m}, 3 \mathrm{H}), 1.30(\mathrm{~d}, 6 \mathrm{H}, J=6.9 \mathrm{~Hz}), 1.17(\mathrm{t}, 3 \mathrm{H}, J=$ $7.0 \mathrm{~Hz}), 1.12(\mathrm{~d}, 6 \mathrm{H}, J=6.8 \mathrm{~Hz}), 0.95(\mathrm{~d}, 6 \mathrm{H}, J=6.9 \mathrm{~Hz})$; minor $E$ isomer [only the most significant signals are listed] $5.68(\mathrm{t}, 1 \mathrm{H}, J=6.6 \mathrm{~Hz}), 4.33$ $(\mathrm{d}, 2 \mathrm{H}, J=6.6 \mathrm{~Hz}), 3.52(\mathrm{q}, 3 \mathrm{H}, J=7.0 \mathrm{~Hz}), 3.15-3.00(\mathrm{~m}, 3 \mathrm{H}) ; \delta_{\mathrm{C}}(75$ $\mathrm{MHz}, \mathrm{CDCl}_{3}$ ) major $Z$ isomer $158.9,147.9,146.3,139.7,132.9,132.3$, 127.3 (2C), 123.2, 120.9 (2C), 113.4 (2C), 69.3, 65.9, 55.1, 34.3, 30.4 (2C), 34.6, 24.5 (2C), 24.4 (2C), 24.1 (2C), 15.3. Anal. Calcd for $\mathrm{C}_{27} \mathrm{H}_{38} \mathrm{O}_{2}$ : C, 82.18; H, 9.71, found: C, 82.01; H, 9.66.

(Z)-1-(4-Ethoxy-1-(4-methoxyphenyl)but-1-en-1-yl)-2-(methoxymethoxy naphthalene $(\mathbf{2} \mathbf{j})$

Yield: 58\%; yellow oil; $\mathrm{R}_{\mathrm{f}}$ : 0.47 (EtOAc/c-hexane: 2/8); IR $\left(\mathrm{v}, \mathrm{cm}^{-1}\right)$ 2939, 1658, 1596, 1509, 1226, 1143, 1099, 837, 803, 749; $\delta_{\mathrm{H}}(300 \mathrm{MHz}$, $\left.\mathrm{CDC1}_{3}\right) 7.90-7.69(\mathrm{~m}, 3 \mathrm{H}), 7.49(\mathrm{~d}, 1 \mathrm{H}, J=9.1 \mathrm{~Hz}), 7.40-7.31(\mathrm{~m}, 2 \mathrm{H})$, $7.21(\mathrm{~d}, 2 \mathrm{H}, J=8.9 \mathrm{~Hz}), 6.76(\mathrm{~d}, 2 \mathrm{H}, J=8.9 \mathrm{~Hz}), 6.48(\mathrm{t}, 1 \mathrm{H}, J=7.2 \mathrm{~Hz})$, $5.20(\mathrm{~d}, 1 \mathrm{H}, J=6.8 \mathrm{~Hz}), 5.12(\mathrm{~d}, 1 \mathrm{H}, J=6.8 \mathrm{~Hz}), 3.74(\mathrm{~s}, 3 \mathrm{H}), 3.49-3.30$ $(\mathrm{m}, 7 \mathrm{H}), 2.25-2.08(\mathrm{~m}, 2 \mathrm{H}), 1.13(\mathrm{t}, 3 \mathrm{H}, J=7.0 \mathrm{~Hz}) ; \delta_{\mathrm{C}}\left(75 \mathrm{MHz}, \mathrm{CDCl}_{3}\right)$ $158.8,151.7,136.1,133.9,133.2,129.9,129.1,128.0,127.2$ (2C), 126.6, $125.7,125.4,124.1,116.7,113.7$ (2C), 94.9, 69.9, 66.1, 56.1, 55.3, 30.8, 27.0, 15.3; MS (APCI+) $m / z$ 393.0 $\left(\mathrm{MH}^{+}\right)$. Anal. Calcd for $\mathrm{C}_{25} \mathrm{H}_{28} \mathrm{O}_{4}: \mathrm{C}$, 76.50; H, 7.19, found: C, 76.34; H, 7.07.

\section{(Z)-(2-(4-ethoxy-1-(4-methoxyphenyl)but-1-en-1-yl)phenyl)(methyl) sulfane $(\mathbf{2 k})$}

Yield: 55\%; brownish oil; $\mathrm{R}_{\mathrm{f}}$ : 0.75 (EtOAc/c-hexane: 2/8); IR ( $\left.\mathrm{v}, \mathrm{cm}^{-1}\right)$ 2972, 1606, 1509, 1434, 1375, 1289, 1245, 1180, 1107, 1035, 828, 793, 742,$633 ; \delta_{\mathrm{H}}\left(300 \mathrm{MHz}, \mathrm{CDC1}_{3}\right) 7.38-7.12(\mathrm{~m}, 5 \mathrm{H}), 7.09(\mathrm{dd}, 1 \mathrm{H}, J=7.4$, $1.3 \mathrm{~Hz}), 6.80(\mathrm{~d}, 2 \mathrm{H}, J=8.9 \mathrm{~Hz}), 6.20(\mathrm{t}, 1 \mathrm{H}, J=7.4 \mathrm{~Hz}), 3.77(\mathrm{~s}, 3 \mathrm{H}), 3.54$ $-3.40(\mathrm{~m}, 4 \mathrm{H}), 2.35(\mathrm{~s}, 3 \mathrm{H}), 2.24(\mathrm{~m}, 2 \mathrm{H}), 1.19(\mathrm{t}, 3 \mathrm{H}, J=7.0 \mathrm{~Hz}) ; \delta_{\mathrm{C}}(75$ $\left.\mathrm{MHz}, \mathrm{CDCl}_{3}\right) 158.9,140.2,138.2$ (2C), 133.2, 130.3, 127.9, 127.4 (2C), $125.3,124.5$ (2C), 113.7 (2C), 70.1, 66.1, 55.3, 30.6, 15.3, 15.3.; MS (APCI+) $m / z, 329.0\left(\mathrm{MH}^{+}\right)$. Anal. Calcd for $\mathrm{C}_{20} \mathrm{H}_{24} \mathrm{O}_{2} \mathrm{~S}: \mathrm{C}, 73.13 ; \mathrm{H}, 7.36$ found: C, 73.00; H, 7.19.

(Z)-1-(4-Ethoxy-1-(4-methoxyphenyl)but-1-en-1-yl)-4-methoxy-2-nitro benzene (2l)

Yield: 65\%; brown oil; $\mathrm{R}_{\mathrm{f}}$ : 0.63 (EtOAc/c-hexane: 4/6); IR $\left(v, \mathrm{~cm}^{-1}\right)$ 2972, 1606, 1509, 1434, 1375, 1289, 1245, 1180, 1107, 1035, 828, 793, 742,$633 ; \delta_{\mathrm{H}}\left(300 \mathrm{MHz}, \mathrm{CDC1}_{3}\right) 7.53(\mathrm{~d}, 1 \mathrm{H}, J=2.4 \mathrm{~Hz}), 7.25(\mathrm{~d}, 1 \mathrm{H}, J=$ $8.4 \mathrm{~Hz}), 7.19(\mathrm{dd}, 1 \mathrm{H}, J=8.4,2.4 \mathrm{~Hz}), 7.12(\mathrm{~d}, 2 \mathrm{H}, J=8.9 \mathrm{~Hz}), 6.78(\mathrm{~d}$,
$2 \mathrm{H}, J=8.9 \mathrm{~Hz}), 6.09(\mathrm{t}, 1 \mathrm{H}, J=7.5 \mathrm{~Hz}), 3.90(\mathrm{~s}, 3 \mathrm{H}), 3.77(\mathrm{~s}, 3 \mathrm{H}), 3.47-$ $3.42(\mathrm{~m}, 4 \mathrm{H}), 2.22(\mathrm{~m}, 2 \mathrm{H}), 1.18(\mathrm{t}, 3 \mathrm{H}, J=7.0 \mathrm{~Hz}) ; \delta_{\mathrm{C}}\left(75 \mathrm{MHz}, \mathrm{CDCl}_{3}\right)$ 159.3, 159.1, 149.7, 138.6, 133.3, 133.7, 127.7 (2C), 127.4, 125.0, 113.8 (2C), 119.8, 109.3, 69.9, 66.3, 55.9, 55.4, 30.8, 15.3; MS (APCI+) $\mathrm{m} / \mathrm{z}$ $358.0\left(\mathrm{MH}^{+}\right)$. Anal. Calcd for $\mathrm{C}_{20} \mathrm{H}_{23} \mathrm{NO}_{5}$ : C, 67.21; H, 6.49; N, 3.92, found: $\mathrm{C}, 67.05 ; \mathrm{H}, 6.30 ; \mathrm{N}, 3.68$.

(Z)-4-Methoxy-2-(methoxymethoxy)-1-(1-(4-methoxyphenyl)oct-1-en-1yl)benzene $(\mathbf{2 m})$

Yield: 72\%; colorless oil; $\mathrm{R}_{\mathrm{f}}$ : 0.58 (EtOAc/c-hexane: 3/7); IR $\left(v, \mathrm{~cm}^{-1}\right)$ $2978,1514,1434,1375,1183,1107,1035,913,828,793,742,650 ; \delta_{\mathrm{H}}$ $\left(300 \mathrm{MHz}, \mathrm{CDC1}_{3}\right)$ only the major $Z$ isomer is described $7.16(\mathrm{~d}, 2 \mathrm{H}, J=$ $8.6 \mathrm{~Hz}), 6.99(\mathrm{~d}, 1 \mathrm{H}, J=8.3 \mathrm{~Hz}), 6.80-6.73(\mathrm{~m}, 3 \mathrm{H}), 6.59(\mathrm{dd}, 1 \mathrm{H}, J=$ $8.3,2.4 \mathrm{~Hz}), 6.07(\mathrm{t}, 1 \mathrm{H}, J=7.3 \mathrm{~Hz}), 4.99(\mathrm{~s}, 2 \mathrm{H}), 3.83(\mathrm{~s}, 3 \mathrm{H}), 3.77(\mathrm{~s}$, $3 \mathrm{H}), 3.25(\mathrm{~s}, 3 \mathrm{H}), 1.99(\mathrm{q}, 2 \mathrm{H}, J=7.3 \mathrm{~Hz}), 1.47-1.14(\mathrm{~m}, 8 \mathrm{H}), 0.85(\mathrm{t}, 3 \mathrm{H}$, $J=6.8 \mathrm{~Hz}) ; \delta_{\mathrm{C}}\left(75 \mathrm{MHz}, \mathrm{CDCl}_{3}\right)$ only the major $Z$ isomer is described $160.1,155.8,158.6,136.9,135.4,132.1,129.3,127.4$ (2C), $122.8,113.5$ (2C), 106.7, 102.3, 94.7, 55.9, 55.5, 55.4, 31.9, 30.1, 29.8, 29.2, 22.8, 14.2; MS (APCI+) $m / z$ 385.0 $\left(\mathrm{MH}^{+}\right)$. Anal. Calcd for $\mathrm{C}_{24} \mathrm{H}_{32} \mathrm{O}_{3}$ : C, 74.97; H, 8.39, found: $\mathrm{C}, 74.89 ; \mathrm{H}, 8.27$.

$$
\text { (2n) }
$$

(Z)-4-Methoxy-1-(1-(4-methoxyphenyl)oct-1-en-1-yl)-2-nitrobenzene

Yield: 52\%; colorless oil; $\mathrm{R}_{\mathrm{f}}$ : 0.64 (EtOAc/c-hexane: 5/5); IR $\left(\mathrm{v}, \mathrm{cm}^{-1}\right)$; $2972,1701,1606,1509,1434,1289,1180,999,802$; [the presence of an inseparable impurity complicates the spectrum] $\delta_{\mathrm{H}}\left(300 \mathrm{MHz}, \mathrm{CDC}_{3}\right) 7.52$ $(\mathrm{d}, 1 \mathrm{H}, J=1.9 \mathrm{~Hz}), 7.20(\mathrm{~d}, 1 \mathrm{H}, J=8.1 \mathrm{~Hz}), 7.17(\mathrm{dd}, 1 \mathrm{H}, J=8.1,1.9 \mathrm{~Hz})$, $7.11(\mathrm{~d}, 2 \mathrm{H}, J=8.5 \mathrm{~Hz}), 6.78(\mathrm{~d}, 2 \mathrm{H}, J=8.5 \mathrm{~Hz}), 3.91(\mathrm{~s}, 3 \mathrm{H}), 3.77(\mathrm{~s}, 3 \mathrm{H})$, $1.95-1.84(\mathrm{q}, 2 \mathrm{H}, J=6.9 \mathrm{~Hz}), 1.43-1.33(\mathrm{~m}, 2 \mathrm{H}), 1.29-1.11(\mathrm{~m}, 4 \mathrm{H}), 0.85$ $(\mathrm{t}, 3 \mathrm{H}, J=6.9 \mathrm{~Hz}) ; \delta_{\mathrm{C}}\left(75 \mathrm{MHz}, \mathrm{CDCl}_{3}\right) 159.2,158.9,149.9,136.7,133.7$, 133.6, 129.5, 127.8 (2C), 127.7, 113.7 (2C), 119.7, 109.1, 56.9, 55.4, 31.8, 30.0, 29.6, 29.1, 22.7, 14.2; MS (APCI+) $\mathrm{m} / \mathrm{z} 370.6\left(\mathrm{MH}^{+}\right)$. (20)

(Z)-1-(3-Ethoxy-1-(3-methoxyphenyl)prop-1-enyl)-2-methoxybenzene

Yield: 32\%; yellow oil; $\mathrm{R}_{\mathrm{f}}$ : 0.23 (EtOAc/c-hexane: 5/95); IR $\left(v, \mathrm{~cm}^{-1}\right)$ 2924, 2834, 1597, 1578, 1482, 1469, 1434, 1373, 1335, 1294, 1244, 1169, 1130, 1096, 1049, 1026, 907, 883, 785, 751, 661, 631; $\delta_{\mathrm{H}}(300 \mathrm{MHz}$, $\mathrm{CDCl}_{3}$ ) [the presence of $Z / E$ isomers in a ratio of $96 / 4$ complicates the spectrum] major $Z$ isomer 7.33 (ddd, $1 \mathrm{H}, J=8.2,7.4,1.9 \mathrm{~Hz}), 7.16(\mathrm{t}, 1 \mathrm{H}, J$ $=8.2 \mathrm{~Hz}), 7.09(\mathrm{dd}, 1 \mathrm{H}, J=7.4,1.9 \mathrm{~Hz}), 6.99(\mathrm{dd}, 1 \mathrm{H}, J=7.4,1.1 \mathrm{~Hz})$, $6.94(\mathrm{~d}, 1 \mathrm{H}, J=8.2 \mathrm{~Hz}), 6.88-6.81(\mathrm{~m}, 2 \mathrm{H}), 6.77(\mathrm{ddd}, 1 \mathrm{H}, J=8.2,2.5$, $1.1 \mathrm{~Hz}), 6.34(\mathrm{t}, 1 \mathrm{H}, J=6.4 \mathrm{~Hz}), 3.93(\mathrm{~d}, 2 \mathrm{H}, J=6.4 \mathrm{~Hz}), 3.76(\mathrm{~s}, 3 \mathrm{H}), 3.70$ $(\mathrm{s}, 3 \mathrm{H}), 3.43(\mathrm{q}, 2 \mathrm{H}, J=7.0 \mathrm{~Hz}), 1.17(\mathrm{t}, 3 \mathrm{H}, J=7.0 \mathrm{~Hz})$; minor $E$ isomer [only the most significant signals are listed] $6.03(\mathrm{t}, 1 \mathrm{H}, J=6.6 \mathrm{~Hz}), 4.15$ $(\mathrm{d}, 2 \mathrm{H}, J=6.6 \mathrm{~Hz}) ; \delta_{\mathrm{C}}\left(75 \mathrm{MHz}, \mathrm{CDCl}_{3}\right)$ only the major $\mathrm{Z}$ isomer is described 159.6, 157.2, 142.8, 139.9, 131.3, 129.1 (2C), 128.0, 127.0, $120.6,119.4,112.7,112.4,111.2,68.8,65.7,55.7,55.3,15.4$; MS (APCI+) $\mathrm{m} / z$ 299.0 $\left(\mathrm{MH}^{+}\right)$. Anal. Calcd for $\mathrm{C}_{19} \mathrm{H}_{22} \mathrm{O}_{3}$ : C, 76.48; H, 7.43, found: C, 76.30; H, 7.26.

(Z)-1-(3-Ethoxy-1-(4-methoxyphenyl)prop-1-en-1-yl)-2-(methoxy methoxy)benzene (2p)

Yield: 68\%; colorless oil; $\mathrm{R}_{\mathrm{f}}$ : 0.34 (EtOAc/c-hexane: 1/9); IR $\left(v, \mathrm{~cm}^{-1}\right)$ 2930, 2149, 2010, 1606, 1510, 1487, 1450, 1373, 1246, 1198, 1180, 1152, $1076,1036,995,906,825,755,729 ; \delta_{\mathrm{H}}\left(300 \mathrm{MHz}, \mathrm{CDC}_{3}\right)$ [the presence of $Z / E$ isomers in a ratio of $9 / 1$ complicates the spectrum] major $Z$ isomer $7.43-7.04(\mathrm{~m}, 6 \mathrm{H}), 6.78(\mathrm{~d}, 2 \mathrm{H}, J=8.9 \mathrm{~Hz}), 6.25(\mathrm{t}, 1 \mathrm{H}, J=6.6 \mathrm{~Hz}), 5.02$ $(\mathrm{s}, 2 \mathrm{H}), 3.95(\mathrm{~d}, 2 \mathrm{H}, J=6.6 \mathrm{~Hz}), 3.77(\mathrm{~s}, 3 \mathrm{H}), 3.43(\mathrm{q}, 2 \mathrm{H}, J=7.0 \mathrm{~Hz}), 3.26$ $(\mathrm{s}, 3 \mathrm{H}), 1.17(\mathrm{t}, 3 \mathrm{H}, J=7.0 \mathrm{~Hz})$; minor $E$ isomer [only the most significant signals are listed] $6.83(\mathrm{~d}, 2 \mathrm{H}, J=8.9 \mathrm{~Hz}), 5.95(\mathrm{t}, 1 \mathrm{H}, J=6.6 \mathrm{~Hz}), 4.88(\mathrm{~s}$, $2 \mathrm{H}), 4.18(\mathrm{~d}, 2 \mathrm{H}, J=6.6 \mathrm{~Hz}), 3.79(\mathrm{~s}, 3 \mathrm{H}), 3.51(\mathrm{q}, 2 \mathrm{H}, J=7.0 \mathrm{~Hz}), 3.16(\mathrm{~s}$, $3 \mathrm{H}) ; \delta_{\mathrm{C}}\left(75 \mathrm{MHz}, \mathrm{CDCl}_{3}\right)$ major $Z$ isomer $158.9,154.7,139.8,133.9$, 131.4, 129.3, 128.9, 127.7 (2C), 124.7, 121.9, 115.2, 113.6 (2C), 94.4, 68.7, $65.6,55.9,55.2,15.3$; minor $E$ isomer [only the most significant signals are listed] 131.2, 130.2, 129.1, 113.2, 94.5, 68.4, 55.8; MS (APCI+) $\mathrm{m} / \mathrm{z} 329.6$ $\left(\mathrm{MH}^{+}\right)$. Anal. Calcd for $\mathrm{C}_{20} \mathrm{H}_{24} \mathrm{O}_{4}: \mathrm{C}, 73.15 ; \mathrm{H}, 7.37$, found: C, 72.97; $\mathrm{H}$, 7.08 .

(Z)-2-(3-Ethoxy-1-(4-methoxyphenyl)prop-1-en-1-yl)-1-(methoxy methoxy)-4-methylbenzene (2q)

Yield: 50\%; yellow oil; $\mathrm{R}_{\mathrm{f}}$ : 0.38 (EtOAc/c-hexane: $\left.2 / 8\right)$; IR $\left(v, \mathrm{~cm}^{-1}\right)$ 2918, 1606, 1511, 1494, 1464, 1292, 1248, 1230, 1199, 1179, 1147, 1098, $1072,1036,1010,985,922,823,806,732 ; \delta_{\mathrm{H}}\left(300 \mathrm{MHz}, \mathrm{CDC}_{3}\right)$ only the major $Z$ isomer is described $7.22(\mathrm{~d}, 2 \mathrm{H}, J=8.9 \mathrm{~Hz}), 7.11(\mathrm{dd}, 1 \mathrm{H}, J=8.4$, $2.2 \mathrm{~Hz}), 7.05(\mathrm{~d}, 1 \mathrm{H}, J=8.4 \mathrm{~Hz}), 6.93(\mathrm{~d}, 1 \mathrm{H}, J=2.2 \mathrm{~Hz}), 6.80(\mathrm{~d}, 2 \mathrm{H}, J=$ $8.9 \mathrm{~Hz}), 6.24(\mathrm{t}, 1 \mathrm{H}, J=6.5 \mathrm{~Hz}), 4.99(\mathrm{~s}, 2 \mathrm{H}), 3.96(\mathrm{~d}, 2 \mathrm{H}, J=6.5 \mathrm{~Hz}), 3.78$ 
(s, 3H), $3.45(\mathrm{q}, 2 \mathrm{H}, J=7.0 \mathrm{~Hz}), 3.27(\mathrm{~s}, 3 \mathrm{H}), 2.30(\mathrm{~s}, 3 \mathrm{H}), 1.19(\mathrm{t}, 3 \mathrm{H}, J=$ $7.0 \mathrm{~Hz}) ; \delta_{\mathrm{C}}\left(75 \mathrm{MHz}, \mathrm{CDCl}_{3}\right)$ only the major $Z$ isomer is described 158.9 , $152.5,139.7,133.9,131.7,131.2,129.3,129.0,127.7$ (2C), 124.5, 115.3, 113.4 (2C), 94.7, 68.7, 65.6, 55.8, 55.2, 20.5, 15.3; MS (ESI+) $\mathrm{m} / z 365.2$ $\left(\mathrm{MNa}^{+}\right.$). Anal. Calcd for $\mathrm{C}_{21} \mathrm{H}_{26} \mathrm{O}_{4}: \mathrm{C}, 73.66 ; \mathrm{H}, 7.65$, found: $\mathrm{C}, 73.37 ; \mathrm{H}$, 7.31 .

(Z)-3-(3-Ethoxy-1-(4-methoxyphenyl)prop-1-en-1-yl)-4-(methoxy methoxy)benzonitrile (2r)

Yield: 52\%; yellow oil; $\mathrm{R}_{\mathrm{f}}$ : 0.53 (EtOAc/c-hexane: 3/7); IR (v, $\left.\mathrm{cm}^{-1}\right)$ 2926, 2227, 1600, 1511, 1491, 1244, 1148, 1078, 1035, 923, 822, 785, 734, 669,$648 ; \delta_{\mathrm{H}}\left(300 \mathrm{MHz}, \mathrm{CDC1}_{3}\right)$ only the major $Z$ isomer is described 7.62 $(\mathrm{dd}, 1 \mathrm{H}, J=8.6,2.2 \mathrm{~Hz}), 7.44(\mathrm{~d}, 1 \mathrm{H}, J=2.2 \mathrm{~Hz}), 7.22(\mathrm{~d}, 1 \mathrm{H}, J=8.6 \mathrm{~Hz})$, $7.13(\mathrm{~d}, 2 \mathrm{H}, J=8.9 \mathrm{~Hz}), 6.80(\mathrm{~d}, 2 \mathrm{H}, J=8.9 \mathrm{~Hz}), 6.28(\mathrm{t}, 1 \mathrm{H}, J=6.6 \mathrm{~Hz})$, 5.09 (s, 2H), $3.87(\mathrm{~d}, 2 \mathrm{H}, J=6.6 \mathrm{~Hz}), 3.78(\mathrm{~s}, 3 \mathrm{H}), 3.42(\mathrm{q}, 2 \mathrm{H}, J=7.0 \mathrm{~Hz})$, $3.22(\mathrm{~s}, 3 \mathrm{H}), 1.18(\mathrm{t}, 3 \mathrm{H}, J=7.0 \mathrm{~Hz}) ; \delta_{\mathrm{C}}\left(75 \mathrm{MHz}, \mathrm{CDCl}_{3}\right)$ only the major $Z$ isomer is described $159.4,158.1,138.1,135.2,133.6,132.8,130.3,127.6$ (2C), 125.9, 119.0, 115.0, 113.8 (2C), 105.2, 94.1, 68.5, 65.9, 56.4, 55.4, 15.4; MS (ESI+) $\mathrm{m} / z, 376.1\left(\mathrm{MNa}^{+}\right)$. Anal. Calcd for $\mathrm{C}_{21} \mathrm{H}_{23} \mathrm{NO}_{4}: \mathrm{C}, 71.37$; H, 6.56; N, 3.96, found: C, 71.09; H, 6.37; N, 3.78.

(Z)-1-(3-Ethoxy-1-(4-methoxyphenyl)prop-1-en-1-yl)-4-methoxy-2(metho xymethoxy)benzene (2s)

Yield: 64\%; brown oil; $\mathrm{R}_{\mathrm{f}}$ : 0.4 (EtOAc/c-hexane: 3/7); IR $\left(v, \mathrm{~cm}^{-1}\right)$ 2835, 1605, 1575, 1510, 1464, 1442, 1395, 1289, 1245, 1216, 1152, 1097, $1071,1036,1004,992,923,825,805,731 ; \delta_{\mathrm{H}}\left(300 \mathrm{MHz}, \mathrm{CDC}_{3}\right)$ [the presence of $Z / E$ isomers in a ratio of $93 / 7$ complicates the spectrum] major $Z$ isomer $7.20(\mathrm{~d}, 2 \mathrm{H}, J=8.9 \mathrm{~Hz}), 7.01(\mathrm{~d}, 1 \mathrm{H}, J=8.4 \mathrm{~Hz}), 6.78(\mathrm{~d}, 2 \mathrm{H}, J=$ $8.9 \mathrm{~Hz}), 6.74(\mathrm{~d}, 1 \mathrm{H}, J=2.5 \mathrm{~Hz}), 6.59(\mathrm{dd}, 1 \mathrm{H}, J=8.4,2.5 \mathrm{~Hz}), 6.21(\mathrm{t}, 1 \mathrm{H}$, $J=6.5 \mathrm{~Hz}), 4.99(\mathrm{~s}, 2 \mathrm{H}), 3.95(\mathrm{~d}, 2 \mathrm{H}, J=6.5 \mathrm{~Hz}), 3.83(\mathrm{~s}, 3 \mathrm{H}), 3.77(\mathrm{~s}$, $3 \mathrm{H}), 3.43(\mathrm{q}, 2 \mathrm{H}, J=7.0 \mathrm{~Hz}), 3.25(\mathrm{~s}, 3 \mathrm{H}), 1.17(\mathrm{t}, 3 \mathrm{H}, J=7.0 \mathrm{~Hz})$; minor $E$ isomer [only the most significant signals are listed] $7.08(\mathrm{~d}, 1 \mathrm{H}, J=8.4$ $\mathrm{Hz}), 6.53(\mathrm{dd}, 1 \mathrm{H}, J=8.4,2.5 \mathrm{~Hz}), 5.91(\mathrm{t}, 1 \mathrm{H}, J=6.5 \mathrm{~Hz}), 4.86(\mathrm{~s}, 2 \mathrm{H})$, $4.14(\mathrm{~d}, 2 \mathrm{H}, J=6.5 \mathrm{~Hz}), 3.79(\mathrm{~s}, 3 \mathrm{H}), 3.14(\mathrm{~s}, 3 \mathrm{H}) ; \delta_{\mathrm{C}}\left(75 \mathrm{MHz}, \mathrm{CDCl}_{3}\right)$ major $Z$ isomer $160.3,158.9,155.6,139.5,134.2,131.7,127.7$ (2C), 124.6 $121.5,113.4$ (2C), 106.5, 101.9, 94.5, 68.8, 65.5, 55.9, 55.3, 55.2, 15.3; minor $E$ isomer [only the most significant signals are listed] $130.1,126.9$, 112.9, 106.1; MS (APCI+) $m / z 359.3\left(\mathrm{MH}^{+}\right)$. Anal. Calcd for $\mathrm{C}_{21} \mathrm{H}_{26} \mathrm{O}_{5}: \mathrm{C}$, 70.37; H, 7.31, found: C, 70.25; H, 7.20

(Z)-2-(3-Ethoxy-1-(4-methoxyphenyl)prop-1-en-1-yl)-4-fluoro-1(methoxy methoxy)benzene (2t)

Yield: 59\%; colorless oil; $\mathrm{R}_{\mathrm{f}}$ : 0.47 (EtOAc/c-hexane: $\left.2 / 8\right)$; IR $\left(\mathrm{v}, \mathrm{cm}^{-1}\right)$ $1512,1485,1286,1248,903,806,792,731,649,634,619 ; \delta_{\mathrm{H}}(300 \mathrm{MHz}$, $\mathrm{CDC}_{3}$ ) [the presence of $Z / E$ isomers in a ratio of $94 / 6$ complicates the spectrum] major $Z$ isomer $7.18(\mathrm{~d}, 2 \mathrm{H}, J=8.8 \mathrm{~Hz}), 7.14-7.07(\mathrm{~m}, 1 \mathrm{H})$, $7.04-6.95(\mathrm{~m}, 1 \mathrm{H}), 6.88-6.83(\mathrm{~m}, 1 \mathrm{H}), 6.80(\mathrm{~d}, 2 \mathrm{H}, J=8.8), 6.25(\mathrm{t}, 1 \mathrm{H}$, $J=6.6 \mathrm{~Hz}), 4.95(\mathrm{~s}, 2 \mathrm{H}), 3.93(\mathrm{~d}, 2 \mathrm{H}, J=6.6 \mathrm{~Hz}), 3.78(\mathrm{~s}, 3 \mathrm{H}), 3.43(\mathrm{q}, 2 \mathrm{H}$, $J=7.0 \mathrm{~Hz}), 3.27(\mathrm{~s}, 3 \mathrm{H}), 1.18(\mathrm{t}, 3 \mathrm{H}, J=7.0 \mathrm{~Hz})$; minor $E$ isomer [only the most significant signals are listed] $5.96(\mathrm{t}, 1 \mathrm{H}, J=6.2 \mathrm{~Hz}), 4.79(\mathrm{~s}, 2 \mathrm{H})$, $4.15(\mathrm{~d}, 2 \mathrm{H}, J=6.5 \mathrm{~Hz}), 3.19(\mathrm{~s}, 3 \mathrm{H}) ; \delta_{\mathrm{C}}\left(75 \mathrm{MHz} \mathrm{CDCl}_{3}\right)$ major $Z$ isomer 158.4, 156.7 (d, J $231 \mathrm{~Hz}), 149.9,137.8,132.3,126.8$ (2C), 124.3, 116.9 (d, $J 22.6 \mathrm{~Hz}), 115.9(\mathrm{~d}, J 8.0 \mathrm{~Hz}), 114.3(\mathrm{~d}, J 22.7 \mathrm{~Hz}), 112.7(2 \mathrm{C}), 94.3,67.7$, 64.9, 55.1, 54.4, 14.4. (one $\mathrm{C}$ missing); $\mathrm{d}_{\mathrm{F}}\left(376 \mathrm{MHz}, \mathrm{CDCl}_{3}\right.$ ) major $Z$ isomer -122.0; MS (APCI+) $m / z 347.6\left(\mathrm{MH}^{+}\right)$. Anal. Calcd for $\mathrm{C}_{20} \mathrm{H}_{23} \mathrm{FO}_{4}$ : C, 69.35; H, 6.69, found: C, 69.32; H, 6.66.

\section{(Z)-1-(3-Ethoxy-1-(4-methoxyphenyl)prop-1-en-1-yl)-2-(methoxy} methoxy)naphthalene (2u)

Yield: 57\%; yellow oil; $\mathrm{R}_{\mathrm{f}}$ : 0.57 (EtOAc/c-hexane: 2/8); IR ( $\left(\mathrm{cm} \mathrm{cm}^{-1}\right)$ 2939, 1658, 1596, 1509, 1487, 1462, 1450, 1373, 1246, 1226, 1143, 1099. $837,803,749,728,648 ; \delta_{\mathrm{H}}\left(300 \mathrm{MHz}, \mathrm{CDC1}_{3}\right)$ only the major $Z$ isomer is described $7.87(\mathrm{~d}, 1 \mathrm{H}, J=9.0 \mathrm{~Hz}), 7.85-7.80(\mathrm{~m}, 1 \mathrm{H}), 7.80-7.71(\mathrm{~m}$, $1 \mathrm{H}), 7.50(\mathrm{~d}, 1 \mathrm{H}, J=9.0 \mathrm{~Hz}), 7.44-7.31(\mathrm{~m}, 2 \mathrm{H}), 7.25(\mathrm{~d}, 2 \mathrm{H}, J=8.4 \mathrm{~Hz})$, $6.78(\mathrm{~d}, 2 \mathrm{H}, J=8.4 \mathrm{~Hz}), 6.59(\mathrm{t}, 1 \mathrm{H}, J=6.2 \mathrm{~Hz}), 5.20(\mathrm{~d}, 1 \mathrm{H}, J=6.9 \mathrm{~Hz})$, $5.14(\mathrm{~d}, 1 \mathrm{H}, J=6.9 \mathrm{~Hz}), 3.87(\mathrm{dd}, 1 \mathrm{H}, J=12.9,6.1 \mathrm{~Hz}), 3.80-3.72(\mathrm{~m}$, $4 \mathrm{H}), 3.38-3.29(\mathrm{~m}, 5 \mathrm{H}), 1.11(\mathrm{t}, 3 \mathrm{H}, J=7.0 \mathrm{~Hz}) ; \delta_{\mathrm{C}}\left(75 \mathrm{MHz}, \mathrm{CDCl}_{3}\right)$ only the major $Z$ isomer is described 159.1, 151.6, 136.3, 133.1, 132.9, 129.8, 129.4, 128.0, 127.4 (2C), 126.8, 126.2, 125.3, 124.2, 116.6, 113.7 (2C), 94.9, 69.1, 65.8, 56.1, 55.2, 27.0, 15.23; MS (APCI+) $\mathrm{m} / z 379.2\left(\mathrm{MH}^{+}\right)$ Anal. Calcd for $\mathrm{C}_{24} \mathrm{H}_{26} \mathrm{O}_{4}$ : C, 76.17; H, 6.92, found: C, 76.01; H, 6.70.

(Z)-3-(3-Ethoxy-1-(3,4,5-trimethoxyphenyl)prop-1-en-1-yl)-4-(methoxy methoxy)benzonitrile (2v)

Yield: $83 \%$; brown oil; $\mathrm{R}_{\mathrm{f}}$ : 0.26 (EtOAc/c-hexane: 3/7); IR $\left(v, \mathrm{~cm}^{-1}\right)$ $2226,1581,1490,1411,1343,1241,1125,1077,923,821 ; \delta_{\mathrm{H}}(300 \mathrm{MHz}$, $\mathrm{CDC1}_{3}$ ) [the presence of $Z / E$ isomers in a ratio of $93 / 7$ complicates the spectrum] major $Z$ isomer $7.62(\mathrm{dd}, 1 \mathrm{H}, J=8.7,2.1 \mathrm{~Hz}), 7.44(\mathrm{~d}, 1 \mathrm{H}, J=$ $2.1 \mathrm{~Hz}), 7.21(\mathrm{~d}, 1 \mathrm{H}, J=8.7 \mathrm{~Hz}), 6.39(\mathrm{~s}, 2 \mathrm{H}), 6.30(\mathrm{t}, 1 \mathrm{H}, J=6.5 \mathrm{~Hz}), 5.09$ $(\mathrm{s}, 2 \mathrm{H}), 3.86(\mathrm{~d}, 2 \mathrm{H}, J=6.5 \mathrm{~Hz}), 3.79(\mathrm{~s}, 3 \mathrm{H}), 3.74(\mathrm{~s}, 6 \mathrm{H}), 3.42(\mathrm{q}, 2 \mathrm{H}, J=$ $7.0 \mathrm{~Hz}), 3.19(\mathrm{~s}, 3 \mathrm{H}), 1.16(\mathrm{t}, 3 \mathrm{H}, J=7.0 \mathrm{~Hz})$; minor $E$ isomer [only the most significant signals are listed] $6.99(\mathrm{t}, 1 \mathrm{H}, J=6.8 \mathrm{~Hz}), 5.00(\mathrm{~s}, 2 \mathrm{H})$, $4.11(\mathrm{~d}, 2 \mathrm{H}, J=6.8 \mathrm{~Hz}), 3.09(\mathrm{~s}, 3 \mathrm{H}) ; \delta_{\mathrm{C}}\left(75 \mathrm{MHz}, \mathrm{CDCl}_{3}\right)$ major $Z$ isomer 157.9, 153.1 (2C), 138.3, 138.0, 136.0, 135.0, 133.8, 129.8, 127.4, 118.8, $114.9,105.1,103.9$ (2C), 93.9, 68.4, 66.1, 60.8, 56.2, 56.1 (2C), 15.2; MS (ESI+) $m / z 436.3\left(\mathrm{MNa}^{+}\right)$. Anal. Calcd for $\mathrm{C}_{23} \mathrm{H}_{27} \mathrm{NO}_{6}: \mathrm{C}, 66.81 ; \mathrm{H}, 6.58 ; \mathrm{N}$, 3.39, found: C, 66.75; H, 6.50; N, 3.29.

(Z)-1-(3-Ethoxy-1-(3,4,5-trimethoxyphenyl)prop-1-en-1-yl)-2-(methoxy methoxy)naphthalene (2w)

Yield: 83\%; brownish oil; $\mathrm{R}_{\mathrm{f}}$ : 0.43 (EtOAc/c-hexane: 1/9); IR $\left(v, \mathrm{~cm}^{-1}\right)$ 2931, 2339, 2136, 2020, 1704, 1664, 1593, 1509, 1467, 1410, 1334, 1238, 1197, 1170, 1153, 1128, 1104, 1084, 1047, 1013, 924, 809, 750, 664, 646; $\delta_{\mathrm{H}}\left(300 \mathrm{MHz}, \mathrm{CDC1}_{3}\right)$ only the major $Z$ isomer is described $7.86(\mathrm{~d}, 1 \mathrm{H}, J=$ $9.2 \mathrm{~Hz}), 7.85-7.71(\mathrm{~m}, 2 \mathrm{H}), 7.49(\mathrm{~d}, 1 \mathrm{H}, J=9.0 \mathrm{~Hz}), 7.43-7.32(\mathrm{~m}, 2 \mathrm{H})$, $6.62(\mathrm{t}, 1 \mathrm{H}, J=6.0 \mathrm{~Hz}), 6.54(\mathrm{~s}, 2 \mathrm{H}), 5.17(\mathrm{~d}, 1 \mathrm{H}, J=6.9 \mathrm{~Hz}), 5.11(\mathrm{~d}, 1 \mathrm{H}$, $J=6.9 \mathrm{~Hz}), 3.89-3.71(\mathrm{~m}, 2 \mathrm{H}), 3.80(\mathrm{~s}, 3 \mathrm{H}), 3.69(\mathrm{~s}, 6 \mathrm{H}), 3.34(\mathrm{~s}, 3 \mathrm{H})$, $3.31(\mathrm{q}, 2 \mathrm{H}, J=7.0 \mathrm{~Hz}), 1.09(\mathrm{t}, 3 \mathrm{H}, J=7.0 \mathrm{~Hz}) ; \delta_{\mathrm{C}}\left(75 \mathrm{MHz}, \mathrm{CDCl}_{3}\right)$ only the major $Z$ isomer is described 150.7 (2C), 136.7, 136.2, 135.7, 132.0, $128.8,128.4,128.1$ (2C), 127.1, 126.3, 125.8, 125.2 (2C), 124.4, 123.3, $122.4,115.6,93.9,68.1,64.9,55.2$ (2C), 20.2, 14.3; MS (ESI+) $m / z 461.3$ $\left(\mathrm{MNa}^{+}\right.$). Anal. Calcd for $\mathrm{C}_{26} \mathrm{H}_{30} \mathrm{O}_{6}: \mathrm{C}, 71.21 ; \mathrm{H}, 6.90$, found: $\mathrm{C}, 71.00 ; \mathrm{H}$, 6.71 .

(Z)-2-Bromo-4-(3-ethoxy-1-(3,4,5-trimethoxyphenyl)prop-1-en-1-yl)-1methoxy -3-(methoxymethoxy)benzene $(\mathbf{2} \mathbf{x})$

Yield: $80 \%$; yellow oil; $\mathrm{R}_{\mathrm{f}}$ : 0.31 (EtOAc/c-hexane: 3/7); IR $\left(v, \mathrm{~cm}^{-1}\right)$ $2935,1581,1462,1415,1285,1239,1126,1006 ; \delta_{\mathrm{H}}\left(300 \mathrm{MHz}, \mathrm{CDC}_{3}\right)$ [the presence of $Z / E$ isomers in a ratio of $91 / 9$ complicates the spectrum] major $Z$ isomer $7.02(\mathrm{~d}, 1 \mathrm{H}, J=8.7 \mathrm{~Hz}), 6.72(\mathrm{~d}, 1 \mathrm{H}, J=8.7 \mathrm{~Hz}), 6.50(\mathrm{~s}$, $2 \mathrm{H}), 6.26(\mathrm{t}, 1 \mathrm{H}, J=6.2 \mathrm{~Hz}), 4.97(\mathrm{~s}, 2 \mathrm{H}), 3.94-3.81(\mathrm{~m}, 2 \mathrm{H}), 3.94(\mathrm{~s}$, $3 \mathrm{H}), 3.81(\mathrm{~s}, 3 \mathrm{H}), 3.76(\mathrm{~s}, 6 \mathrm{H}), 3.49-3.43(\mathrm{~m}, 5 \mathrm{H}), 1.19(\mathrm{t}, 3 \mathrm{H}, J=7.0$ $\mathrm{Hz}$ ); minor $E$ isomer [only the most significant signals are listed] 7.06 (d, $1 \mathrm{H}, J=8.7 \mathrm{~Hz}), 6.65(\mathrm{~d}, 1 \mathrm{H}, J=8.7 \mathrm{~Hz}), 6.44(\mathrm{~s}, 2 \mathrm{H}), 6.05(\mathrm{t}, 1 \mathrm{H}, J=6.2$ $\mathrm{Hz}), 5.00(\mathrm{~s}, 2 \mathrm{H}), 4.11(\mathrm{~d}, 2 \mathrm{H}, J=6.2 \mathrm{~Hz}), 3.78(\mathrm{~s}, 6 \mathrm{H}), 3.52(\mathrm{~s}, 3 \mathrm{H}) ; \delta_{\mathrm{C}}(75$ $\mathrm{MHz}, \mathrm{CDCl}_{3}$ ) major $Z$ isomer $156.9,153.6,153.1$ (2C), 138.9, 138.0, 136.9, 130.8, 127.2, 126.3, 107.7, 107.4, 104.6 (2C), 99.1, 69.0, 66.3, 61.0, $57.9,56.6,56.3$ (2C), 15.4; minor $E$ isomer [only the most significant signals are listed] 128.9, 106.7, 99.4, 68.2, 66.1, 61.0; MS (ESI+) $\mathrm{m} / \mathrm{z} 520.6$ $\left(\mathrm{MNa}^{+}\right)$. Anal. Calcd for $\mathrm{C}_{23} \mathrm{H}_{29} \mathrm{BrO}_{7}$ : C, 55.54; H, 5.88, found: $\mathrm{C}, 55.27 ; \mathrm{H}$, 5.36 .

(Z)-1-(3-ethoxy-1-(p-tolyl)prop-1-en-1-yl)-2-(methoxymethoxy) naphthalene (2y)

Yield: $85 \%$; pale yellow oil; $\mathrm{R}_{\mathrm{f}}$ : 0.43 (EtOAc/c-hexane: 1/9); IR ( $v$, $\left.\mathrm{cm}^{-1}\right)$ 2931, 2339, 2136, 2020, 1704, 1664, 1593, 1509, 1467, 1410, 1334, 1238, 1197, 1170, 1153, 1128, 1104, 1084, 1047, 1013, 924, 809, 750, 664, $646 ; \delta_{\mathrm{H}}\left(300 \mathrm{MHz}, \mathrm{CDC1}_{3}\right)$ [the presence of $Z / E$ isomers in a ratio of $97 / 3$ complicates the spectrum] major $Z$ isomer $7.86(\mathrm{~d}, 1 \mathrm{H}, J=9.1 \mathrm{~Hz}), 7.84-$ $7.78(\mathrm{~m}, 1 \mathrm{H}), 7.75-7.69(\mathrm{~m}, 1 \mathrm{H}), 7.48(\mathrm{~d}, 1 \mathrm{H}, J=9.0 \mathrm{~Hz}), 7.39-7.32(\mathrm{~m}$, $2 \mathrm{H}), 7.18(\mathrm{~d}, 2 \mathrm{H}, J=8.0 \mathrm{~Hz}), 7.02(\mathrm{~d}, 2 \mathrm{H}, J=8.0 \mathrm{~Hz}), 6.62(\mathrm{t}, 1 \mathrm{H}, J=6.0$ $\mathrm{Hz}), 5.17(\mathrm{~d}, 1 \mathrm{H}, J=6.9 \mathrm{~Hz}), 5.11(\mathrm{~d}, 1 \mathrm{H}, J=6.9 \mathrm{~Hz}), 3.85(\mathrm{dd}, 1 \mathrm{H}, J=$ $13.0,6.0 \mathrm{~Hz}), 3.74(\mathrm{dd}, 1 \mathrm{H}, J=13.0,6.0 \mathrm{~Hz}), 3.33(\mathrm{~s}, 3 \mathrm{H}), 3.31$ (q, 2H, $J=$ $7.0 \mathrm{~Hz}), 2.28(\mathrm{~s}, 3 \mathrm{H}), 1.09(\mathrm{t}, 3 \mathrm{H}, J=7.0 \mathrm{~Hz})$; minor $E$ isomer [only the most significant signals are listed] $5.89(\mathrm{t}, 1 \mathrm{H}, J=6.0 \mathrm{~Hz}), 4.39(\mathrm{~d}, 2 \mathrm{H}, J=$ $6.0 \mathrm{~Hz}) ; \delta_{\mathrm{C}}\left(75 \mathrm{MHz}, \mathrm{CDCl}_{3}\right)$ major $Z$ isomer $150.7,136.7,136.2,135.7$, $132.0,128.8,128.4,128.1$ (2C), 127.1, 126.3, 125.8, 125.2 (2C), 124.4, 123.3, 122.4, 115.6, 93.9, 68.1, 64.9, 55.2, 20.2, 14.3; MS (ESI+) $\mathrm{m} / z .385 .3$ $\left(\mathrm{MNa}^{+}\right.$). Anal. Calcd for $\mathrm{C}_{24} \mathrm{H}_{26} \mathrm{O}_{3}$ : C, 79.53; H, 7.23, found: C, 79.39; H, 7.10.

(Z)-1-(3-Ethoxy-1-(p-tolyl)prop-1-en-1-yl)-4-methoxy-2-(methoxy methoxy)benzene $(\mathbf{2 z})$

Yield: 94\%; brown oil; $\mathrm{R}_{\mathrm{f}}$ : 0.66 (EtOAc/c-hexane: 2/8); IR $\left(v, \mathrm{~cm}^{-1}\right)$ 2973, 2362, 1607, 1576, 1509, 1466, 1441, 1396, 1374, 1298, 1248, 1217, $1153,1118,1097,1072,1039,1005,924,831,810,729,629 ; \delta_{\mathrm{H}}(300$ $\mathrm{MHz}, \mathrm{CDC1}_{3}$ ) [the presence of $\mathrm{Z} / E$ isomers in a ratio of $91 / 9$ complicates the spectrum] major $Z$ isomer $7.17(\mathrm{~d}, 2 \mathrm{H}, J=8.2 \mathrm{~Hz}), 7.05(\mathrm{~d}, 2 \mathrm{H}, J=8.2$ $\mathrm{Hz}), 7.01(\mathrm{~d}, 1 \mathrm{H}, J=8.4 \mathrm{~Hz}), 6.75(\mathrm{~d}, 1 \mathrm{H}, J=2.3 \mathrm{~Hz}), 6.59(\mathrm{dd}, 1 \mathrm{H}, J=$ $8.4,2.3 \mathrm{~Hz}), 6.27(\mathrm{t}, 1 \mathrm{H}, J=6.5 \mathrm{~Hz}), 4.99(\mathrm{~s}, 2 \mathrm{H}), 3.97(\mathrm{~d}, 2 \mathrm{H}, J=6.5 \mathrm{~Hz})$, $3.83(\mathrm{~s}, 3 \mathrm{H}), 3.44(\mathrm{q}, 2 \mathrm{H}, J=7.0 \mathrm{~Hz}), 3.25(\mathrm{~s}, 3 \mathrm{H}), 2.31(\mathrm{~s}, 3 \mathrm{H}), 1.18(\mathrm{t}$, $3 \mathrm{H}, J=7.0 \mathrm{~Hz})$; minor $E$ isomer [only the most significant signals are listed] $6.53(\mathrm{dd}, 1 \mathrm{H}, J=8.4,2.4 \mathrm{~Hz}), 5.95(\mathrm{t}, 1 \mathrm{H}, J=6.6 \mathrm{~Hz}), 4.85(\mathrm{~s}, 2 \mathrm{H})$, $4.15(\mathrm{~d}, 2 \mathrm{H}, J=6.6 \mathrm{~Hz}), 3.79(\mathrm{~s}, 3 \mathrm{H}), 3.14(\mathrm{~s}, 3 \mathrm{H}), 2.33(\mathrm{~s}, 3 \mathrm{H}) ; \delta_{\mathrm{C}}(75$ 
$\left.\mathrm{MHz}, \mathrm{CDCl}_{3}\right)$ 160.4, 155.8, 140.0, 138.9, 137.0, 132.0, 128.9 (2C), 126.6 (2C), 125.8, 121.7, 106.6, 102.1, 94.7, 68.9, 65.7, 56.0, 55.5, 21.2, 15.4; MS (ESI+) $m / z 365\left(\mathrm{MNa}^{+}\right)$. Anal. Calcd for $\mathrm{C}_{21} \mathrm{H}_{26} \mathrm{O}_{4}: \mathrm{C}, 73.66 ; \mathrm{H}, 7.65$, found: $\mathrm{C}, 73.54 ; \mathrm{H}, 7.54$

(Z)-1-(4-Ethoxy-1-(4-methoxyphenyl)but-1-en-1-yl)-2-(methoxy methoxy)benzene $(\mathbf{5})$

Yield: 71\%; yellow oil; $\mathrm{R}_{\mathrm{f}}$ : 0.76 (EtOAc/c-hexane: 8/2); IR $\left(v, \mathrm{~cm}^{-1}\right)$ 2939, 1658, 1509, 1487, 1462, 1450, 1373, 1226, 1143, 837, 803, 749, 648; $\delta_{\mathrm{H}}\left(300 \mathrm{MHz}, \mathrm{CDC1}_{3}\right)$ [the presence of $\mathrm{Z} / \mathrm{E}$ isomers in a ratio of $95 / 5$ complicates the spectrum] major $\mathrm{Z}$ isomer $7.32-7.25(\mathrm{~m}, 1 \mathrm{H}), 7.18(\mathrm{~d}, 2 \mathrm{H}$, $J=8.7 \mathrm{~Hz}), 7.15-7.10(\mathrm{~m}, 2 \mathrm{H}), 7.08-7.00(\mathrm{~m}, 1 \mathrm{H}), 6.79(\mathrm{~d}, 2 \mathrm{H}, J=8.7$ $\mathrm{Hz}), 6.16(\mathrm{t}, 1 \mathrm{H}, J=7.3 \mathrm{~Hz}), 5.03(\mathrm{~s}, 2 \mathrm{H}), 3.77(\mathrm{~s}, 3 \mathrm{H}), 3.51-3.39(\mathrm{~m}$, $4 \mathrm{H}), 3.27(\mathrm{~s}, 3 \mathrm{H}), 2.30(\mathrm{q}, 2 \mathrm{H}, J=7.1 \mathrm{~Hz}), 1.19(\mathrm{t}, 3 \mathrm{H}, J=7.1 \mathrm{~Hz})$; minor $\mathrm{E}$ isomer [only the most significant signals are listed] $5.90 \mathrm{tt}, 1 \mathrm{H}, J=7.4$ $\mathrm{Hz}), 4.98(\mathrm{~s}, 2 \mathrm{H}), 2.66(\mathrm{q}, 2 \mathrm{H}, J=7.1 \mathrm{~Hz}) ; \delta_{\mathrm{C}}\left(75 \mathrm{MHz}, \mathrm{CDCl}_{3}\right)$ major $Z$ isomer $158.7,154.8,139.1,134.6,131.7,130.0,128.7,127.5$ (2C), 124.5, $121.9,115.3,113.5$ (2C), 94.5, 70.3, 66.2, 55.9, 55.3, 30.8, 15.3; MS (APCI+) $m / z 343.5\left(\mathrm{MH}^{+}\right)$. Anal. Calcd for $\mathrm{C}_{21} \mathrm{H}_{26} \mathrm{O}_{4}: \mathrm{C}, 73.66 ; \mathrm{H}, 7.65$, found: C, 73.27; H, 7.39.

General procedure for PTSA assisted cyclisation: An ethanolic (8 $\mathrm{mL})$ solution of trisubstituted olefin $2(0.5 \mathrm{mmol})$ and PTSA $(171 \mathrm{mg}, 1.5$ mmol) was refluxed for $1 \mathrm{~h}$. After cooling to room temperature, water was added $(10 \mathrm{~mL})$ and the media was extracted with EtOAc $(3 \times 10 \mathrm{~mL})$. The organic layers where then washed with brine, dried over $\mathrm{Na}_{2} \mathrm{SO}_{3}$, concentrated and the crude product was purified by column flash chromatography on silica gel.

\section{4-(4-Methoxyphenyl)-2H-chromene $(\mathbf{1 a})^{[10]}$}

Yield: $100 \%$; yellow oil; $\mathrm{R}_{\mathrm{f}}(30 \% \mathrm{AcOEt} /$ Cyclohexane) 0.58 ; IR ( $\mathrm{v}$, $\left.\mathrm{cm}^{-1}\right) 2040,1609,1226,1143,1099,1029,994,907,618 ; \delta_{\mathrm{H}}(300 \mathrm{MHz}$, $\left.\mathrm{CDC1}_{3}\right) 7.27(\mathrm{dd}, 2 \mathrm{H}, J=11.1,3.5 \mathrm{~Hz}), 7.16(\mathrm{td}, 1 \mathrm{H}, J=8.1,1.6 \mathrm{~Hz}), 7.03$ $(\mathrm{dd}, 1 \mathrm{H}, J=7.7,1.5 \mathrm{~Hz}), 6.93(\mathrm{~d}, 2 \mathrm{H}, J=8.8 \mathrm{~Hz}), 6.97-6.80(\mathrm{~m}, 2 \mathrm{H})$, $5.76(\mathrm{t}, 1 \mathrm{H}, J=4.0 \mathrm{~Hz}), 4.84(\mathrm{~d}, 2 \mathrm{H}, J=4.0 \mathrm{~Hz}), 3.85(\mathrm{~s}, 3 \mathrm{H}) ; \delta_{\mathrm{C}}(75 \mathrm{MHz}$ $\left.\mathrm{CDCl}_{3}\right)$ 159.3, 154.8, 136.7, 130.7, 129.7 (2C), 129.2, 125.9, 123.9, 121.1, 119.2, 116.2, $113.8(2 \mathrm{C}), 65.3,55.3$; MS (APCI+) $\mathrm{m} / \mathrm{z} 239\left(\mathrm{MH}^{+}\right)$.

\section{4-(4-Methoxyphenyl)-6-methyl-2H-chromene (1b)}

Yield: 52\%; yellow oil; $\mathrm{R}_{\mathrm{f}}$ : 0.75 (EtOAc/c-hexane: 3/7); IR $\left(v, \mathrm{~cm}^{-1}\right)$ 2928, 2040, 1609, 1511, 1490, 1462, 1342, 1293, 1247, 1229, 1177, 1125, $1035,838,805,612 ; \delta_{\mathrm{H}}\left(300 \mathrm{MHz}, \mathrm{CDC}_{3}\right) 7.26(\mathrm{~d}, 2 \mathrm{H}, J=8.9 \mathrm{~Hz}), 6.99-$ $6.90(\mathrm{~m}, 3 \mathrm{H}), 6.80(\mathrm{~d}, 2 \mathrm{H}, J=8.9 \mathrm{~Hz}), 5.75(\mathrm{t}, 1 \mathrm{H}, J=3.9 \mathrm{~Hz}), 4.79(\mathrm{~d}, 2 \mathrm{H}$, $J=3.9 \mathrm{~Hz}), 3.86(\mathrm{~s}, 3 \mathrm{H}), 2.20(\mathrm{~s}, 3 \mathrm{H}) ; \delta_{\mathrm{C}}\left(75 \mathrm{MHz}, \mathrm{CDCl}_{3}\right) 158.4,139.9$, $135.9,129.5,128.9$ (2C), 128.7, 125.4, 122.9, 118.5, 115.0, 112.9 (2C), 109.6, 64.3, 54.5, 19.9; MS (APCI+) $\mathrm{m} / z 253.1\left(\mathrm{MH}^{+}\right)$. Anal. Calcd for $\mathrm{C}_{17} \mathrm{H}_{16} \mathrm{O}_{2}: \mathrm{C}, 80.93$; H, 6.39, found: $\mathrm{C}, 80.74 ; \mathrm{H}, 6.28$.

\section{4-(4-Methoxyphenyl)-2H-chromene-6-carbonitrile (1c)}

Yield: 59\%; yellow oil; $\mathrm{R}_{\mathrm{f}}$ : 0.7 (EtOAc/c-hexane: 3/7); IR ( $\left.\mathrm{v}, \mathrm{cm}^{-1}\right)$ 2935, 2224, 1609, 1512, 1488, 1344, 1243, 1177, 1122, 1061, 1034, 904, $838,809,729,649 ; \delta_{\mathrm{H}}\left(300 \mathrm{MHz}, \mathrm{CDC}_{3}\right) 7.42(\mathrm{dd}, 1 \mathrm{H}, J=8.4,2.0 \mathrm{~Hz})$, $7.29(\mathrm{~d}, 1 \mathrm{H}, J=2.0 \mathrm{~Hz}), 7.22(\mathrm{~d}, 2 \mathrm{H}, J=8.7 \mathrm{~Hz}), 6.96(\mathrm{~d}, 2 \mathrm{H}, J=8.7 \mathrm{~Hz})$, $6.90(\mathrm{~d}, 1 \mathrm{H}, J=8.4 \mathrm{~Hz}), 5.79(\mathrm{t}, 1 \mathrm{H}, J=3.8 \mathrm{~Hz}), 4.97(\mathrm{~d}, 2 \mathrm{H}, J=3.8 \mathrm{~Hz})$, $3.86(\mathrm{~s}, 3 \mathrm{H}) ; \delta_{\mathrm{C}}\left(75 \mathrm{MHz}, \mathrm{CDCl}_{3}\right) 158.4,139.9,135.9,129.5,128.9$ (2C), 128.7, 125.4, 122.9, 118.5, 115.0, 112.9 (2C), 109.6, 64.3, 54.5, 19.9; MS (APCI+) $m / z, 264.1\left(\mathrm{MH}^{+}\right)$. Anal. Calcd for $\mathrm{C}_{17} \mathrm{H}_{13} \mathrm{NO}_{2}: \mathrm{C}, 77.55 ; \mathrm{H}, 4.98$; N, 5.32, found: C, 77.41; H, 4.79; N, 5.12.

\section{7-Methoxy-4-(4-methoxyphenyl)-2H-chromene (1d)}

Yield: 68\%; colorless oil; $\mathrm{R}_{\mathrm{f}}$ : 0.4 (EtOAc/c-hexane: $\left.2 / 8\right)$; IR $\left(v, \mathrm{~cm}^{-1}\right)$ 2020, 1704, 1636, 803, 781, 749, 728, 698, 612; $\delta_{\mathrm{H}}\left(300 \mathrm{MHz}, \mathrm{CDC}_{3}\right)$ $7.26(\mathrm{~d}, 2 \mathrm{H}, J 8.8 \mathrm{~Hz}), 6.96-6.89(\mathrm{~m}, 3 \mathrm{H}), 6.48-6.39(\mathrm{~m}, 2 \mathrm{H}), 5.60(\mathrm{t}$, $1 \mathrm{H}, J=4.0 \mathrm{~Hz}), 4.79(\mathrm{~d}, 2 \mathrm{H}, J=4.0 \mathrm{~Hz}), 3.83(\mathrm{~s}, 3 \mathrm{H}), 3.78(\mathrm{~s}, 3 \mathrm{H}) ; \delta_{\mathrm{C}}(75$ $\left.\mathrm{MHz}, \mathrm{CDCl}_{3}\right) 160.5,159.3,156.2,136.5,130.9,130.0,126.8,117.2,116.3$, $113.8,106.9,102.0,65.6,55.4,55.3$ (2C missing); MS (ESI+) $\mathrm{m} / z 269.2$ $\left(\mathrm{MH}^{+}\right)$. The spectral data above are in accordance with the literature data ${ }^{[7]}$.

\section{6-Fluoro-4-(4-methoxyphenyl)-2H-chromene (1e)}

Yield: 61\%; yellow oil; $\mathrm{R}_{\mathrm{f}}$ : 0.45 (EtOAc/c-hexane: 1/9); IR $\left(v, \mathrm{~cm}^{-1}\right)$ $1512,1485,1248,903,806,731,649,619 ; \delta_{\mathrm{H}}\left(300 \mathrm{MHz}, \mathrm{CDC}_{3}\right) 7.26(\mathrm{~d}$, $2 \mathrm{H}, J=8.4 \mathrm{~Hz}), 6.94(\mathrm{~d}, 2 \mathrm{H}, J=8.4 \mathrm{~Hz}), 6.86-6.69(\mathrm{~m}, 3 \mathrm{H}), 5.82(\mathrm{t}, 1 \mathrm{H}$, $J=3.9 \mathrm{~Hz}), 4.80(\mathrm{~d}, 2 \mathrm{H}, J=3.9 \mathrm{~Hz}), 3.85(\mathrm{~s}, 3 \mathrm{H}) ; \delta_{\mathrm{C}}\left(75 \mathrm{MHz}, \mathrm{CDCl}_{3}\right)$ $159.7,159.1,153.4$ (d, $J 383.8 \mathrm{~Hz}), 136.4,130.2,129.8$ (2C), 125.3, 120.5, $117.1(\mathrm{~d}, J 8.1 \mathrm{~Hz}), 115.3(\mathrm{~d}, J 23.4 \mathrm{~Hz}), 114.1(2 \mathrm{C}), 112.5(\mathrm{~d}, J 24.8 \mathrm{~Hz})$, 65.5, 55.5, (1C missing); $\mathrm{d}_{\mathrm{F}}\left(175 \mathrm{MHz}, \mathrm{CDCl}_{3}\right)-122.3(\mathrm{td}, J$ 9.3, $6.6 \mathrm{~Hz})$;
MS (APCI+) $m / z 257.0\left(\mathrm{MH}^{+}\right)$. Anal. Calcd for $\mathrm{C}_{16} \mathrm{H}_{13} \mathrm{FO}_{2}: \mathrm{C}, 74.99 ; \mathrm{H}$, 5.11, found: $\mathrm{C}, 74.78 ; \mathrm{H}, 4.99$.

\section{1-(4-Methoxyphenyl)-3H-benzo[f]chromene (1f)}

Yield: 78\%; yellow oil; $\mathrm{R}_{\mathrm{f}}$ : 0.75 (EtOAc/c-hexane: 1/8); IR $\left(v, \mathrm{~cm}^{-1}\right)$ 1658, 1596, 1509, 1462, 1246, 1226, 1143, 1099, 1029, 994, 907, 837, 803, 781, 749, 728, 648, 615; $\delta_{\mathrm{H}}\left(300 \mathrm{MHz}, \mathrm{CDC1}_{3}\right) 7.77(\mathrm{~d}, 2 \mathrm{H}, J=8.5 \mathrm{~Hz})$, $7.34-7.06(\mathrm{~m}, 6 \mathrm{H}), 6.88(\mathrm{~d}, 2 \mathrm{H}, J=8.5 \mathrm{~Hz}), 5.96(\mathrm{t}, 1 \mathrm{H}, J=4.9 \mathrm{~Hz}), 4.68$ $(\mathrm{d}, 2 \mathrm{H}, J=4.9 \mathrm{~Hz}), 3.84(\mathrm{~s}, 3 \mathrm{H}) ; \delta_{\mathrm{C}}\left(75 \mathrm{MHz}, \mathrm{CDCl}_{3}\right) 159.0,155.1,137.6$ 133.6, 130.35, 130.3, 130.1, 128.8 (2C), 128.4, 126.6, 125.2, 123.3, 119.1, 117.75, 117.7, 113.8 (2C), 64.5, 55.3; MS (ESI+) $m / z 311.02\left(\mathrm{MNa}^{+}\right)$. Anal. Calcd for $\mathrm{C}_{20} \mathrm{H}_{16} \mathrm{O}_{2}$ : C, 83.31; H, 5.59, found: $\mathrm{C}, 83.00 ; \mathrm{H}, 5.32$. The spectral data above are in accordance with the literature data ${ }^{[10]}$.

\section{1-(3,4,5-Trimethoxyphenyl)-2H-chromene-6-carbonitrile (1g)}

Yield: 77\%; colorless oil; $\mathrm{R}_{\mathrm{f}}$ : 0.51 (EtOAc/c-hexane: 4/6); IR $\left(\mathrm{v}, \mathrm{cm}^{-1}\right)$ 2224, 1580, 1507, 1488, 1409, 1355, 1240, 1123, 1063, 1005, 943, 825, $733 ; \delta_{\mathrm{H}}\left(300 \mathrm{MHz}, \mathrm{CDC1}_{3}\right) 7.43(\mathrm{dd}, 1 \mathrm{H}, J=8.4,2.0 \mathrm{~Hz}), 7.30(\mathrm{~d}, 1 \mathrm{H}, J=$ $2.0 \mathrm{~Hz}), 6.91(\mathrm{~d}, 1 \mathrm{H}, J=8.4 \mathrm{~Hz}), 6.48(\mathrm{~s}, 2 \mathrm{H}), 5.83(\mathrm{t}, 1 \mathrm{H}, J=3.7 \mathrm{~Hz}), 4.98$ $(\mathrm{d}, 2 \mathrm{H}, J=3.7 \mathrm{~Hz}), 3.91(\mathrm{~s}, 3 \mathrm{H}), 3.86(\mathrm{~s}, 6 \mathrm{H}) ; \delta_{\mathrm{C}}\left(75 \mathrm{MHz}, \mathrm{CDCl}_{3}\right) 158.4$, 153.6 (2C), 138.3, 135.7, 133.6, 132.6, 129.9, 124.3, 121.1, 119.3, 117.4, 105.7 (2C), 104.6, 66.1, 61.1, $56.4(2 \mathrm{C})$; MS (APCI+) $\mathrm{m} / z$ 324.3 $\left(\mathrm{MH}^{+}\right)$ Anal. Calcd for $\mathrm{C}_{19} \mathrm{H}_{17} \mathrm{NO}_{4}$ : C, 70.58; H, 5.30; N, 4.33, found: C, 70.25; H, $5.18 ; \mathrm{N}, 4.29$.

\section{1-(3,4,5-Trimethoxyphenyl)-3H-benzo[f]chromene (1h)}

Yield: 91\%; colorless oil; $\mathrm{R}_{\mathrm{f}}$ : $0.72\left(\right.$ EtOAc/c-hexane: 3/7); IR $\left(\mathrm{v}, \mathrm{cm}^{-1}\right)$ $2930,1580,1508,1464,1414,1343,1232,1126,997,815,732 ; \delta_{\mathrm{H}}(300$ $\left.\mathrm{MHz}_{\mathrm{CDC1}}\right) 7.74(\mathrm{~m}, 2 \mathrm{H}), 7.25-7.06(\mathrm{~m}, 4 \mathrm{H}), 6.40(\mathrm{~s}, 2 \mathrm{H}), 6.01(\mathrm{t}, 1 \mathrm{H}$, $J=4.8 \mathrm{~Hz}), 4.69(\mathrm{~d}, 2 \mathrm{H}, J=4.8 \mathrm{~Hz}), 3.89(\mathrm{~s}, 3 \mathrm{H}), 3.70(\mathrm{~s}, 6 \mathrm{H}) ; \delta_{\mathrm{C}}(75$ $\mathrm{MHz} \mathrm{CDCl}_{3}$ ) 154.1, 152.3 (3C), 137.2, 135.9, 129.71, 129.4, 129.1, 127.4, 125.6, 124.4, 122.5, 118.8, 116.8, 116.6, 104.3 (2C), 63.6, 60.2, 55.3 (2C); MS (APCI+) $m / z .349 .3\left(\mathrm{MH}^{+}\right)$. Anal. Calcd for $\mathrm{C}_{22} \mathrm{H}_{20} \mathrm{O}_{4}: \mathrm{C}, 75.84 ; \mathrm{H}$, 5.79, found: $\mathrm{C}, 75.75 ; \mathrm{H}, 5.70$.

\section{8-Bromo-7-methoxy-4-(3,4,5-trimethoxyphenyl)-2H-chromene (1i)}

Yield: 95\%; colorless oil; $\mathrm{R}_{\mathrm{f}}$ : 0.24 (EtOAc/c-hexane: 3/7); IR ( $\left.\mathrm{v}, \mathrm{cm}^{-1}\right)$ $3663,3324,2974,2224,2012,1608,1511,1487,1442,730,700 ; \delta_{\mathrm{H}}(300$ $\left.\mathrm{MHz}, \mathrm{CDC1}_{3}\right) 6.96(\mathrm{~d}, 1 \mathrm{H}, J=8.6 \mathrm{~Hz}), 6.52(\mathrm{~s}, 2 \mathrm{H}), 6.43(\mathrm{~d}, 1 \mathrm{H}, J=8.6$ $\mathrm{Hz}), 5.70(\mathrm{t}, 1 \mathrm{H}, J=3.9 \mathrm{~Hz}), 4.93(\mathrm{~d}, 2 \mathrm{H}, J=3.9 \mathrm{~Hz}), 3.88(\mathrm{~s}, 6 \mathrm{H}), 3.84(\mathrm{~s}$, $6 \mathrm{H}) ; \delta_{\mathrm{C}}\left(75 \mathrm{MHz}, \mathrm{CDCl}_{3}\right)$ 157.0, $153.3(2 \mathrm{C}), 153.2,136.9,133.8,125.3$, $119.6,118.3,117.5,105.8(2 \mathrm{C}), 104.1,100.3,66.3,61.0,56.5,56.3(2 \mathrm{C})$; MS (APCI+) $m / z$ 407.2, $409.1\left(\mathrm{MH}^{+}\right)$. Anal. Calcd for $\mathrm{C}_{19} \mathrm{H}_{19} \mathrm{BrO}_{5}: \mathrm{C}$, 56.03 ; H, 4.70, found: C, 55.80; H, 4.63 .

\section{1-p-Tolyl-3H-benzo[f]chromene $(\mathbf{1} \mathbf{j})$}

Yield: 74\%; colorless oil; $\mathrm{R}_{\mathrm{f}}$ : 0.68 (EtOAc/c-hexane: 5/95); IR ( $v, \mathrm{~cm}^{-1}$ ) : 2934, 1672, 1579, 1499, 1410, 1374, 1335, 1235, 1168, 1124, 1031, 1007, $910,835,789,730 ; \delta_{\mathrm{H}}\left(300 \mathrm{MHz}, \mathrm{CDC1}_{3}\right) 7.74(\mathrm{~d}, 2 \mathrm{H}, J=8.6 \mathrm{~Hz}), 7.26-$ $7.04(\mathrm{~m}, 8 \mathrm{H}), 5.96(\mathrm{t}, 1 \mathrm{H}, J=4.9 \mathrm{~Hz}), 4.68(\mathrm{~d}, 2 \mathrm{H}, J=4.9 \mathrm{~Hz}), 2.38(\mathrm{~s}$, $3 \mathrm{H}) ; \delta_{\mathrm{C}}\left(75 \mathrm{MHz}, \mathrm{CDCl}_{3}\right) 155.1,138.5,138.0,137.0,130.5,130.3,130.2$, $128.5,129.3$ (2C), 127.8 (2C), 126.8, 125.3, 123.4, 119.8, 117.9, 117.8, 64.7, 21.4; MS (APCI+) $\mathrm{m} / z$ 273.4 $\left(\mathrm{MH}^{+}\right)$. Anal. Calcd for $\mathrm{C}_{20} \mathrm{H}_{16} \mathrm{O}: \mathrm{C}$, 88.20 ; H, 5.92, found: C, 87.79; H, 5.62 .

\section{7-Methoxy-4-p-tolyl-2H-chromene (1k)}

Yield: 79\%; colorless oil; $\mathrm{R}_{\mathrm{f}}$ : 0.55 (EtOAc/c-hexane: 1/9); IR $\left(v, \mathrm{~cm}^{-1}\right)$ 2955, 1612, 1567, 1502, 1376, 1346, 1309, 1281, 1195, 1160, 1128, 1115, $1034,973,805,741 ; \delta_{\mathrm{H}}\left(300 \mathrm{MHz}, \mathrm{CDC1}_{3}\right) 7.23-7.19(\mathrm{~m}, 4 \mathrm{H}), 6.95(\mathrm{~d}$, $1 \mathrm{H}, J=8.5 \mathrm{~Hz}), 6.50(\mathrm{~d}, 1 \mathrm{H}, J=2.4 \mathrm{~Hz}), 6.42(\mathrm{dd}, 1 \mathrm{H}, J=8.5,2.4 \mathrm{~Hz})$, $5.64(\mathrm{t}, 1 \mathrm{H}, J=3.9 \mathrm{~Hz}), 4.83(\mathrm{~d}, 2 \mathrm{H}, J=3.9 \mathrm{~Hz}), 3.80(\mathrm{~s}, 3 \mathrm{H}), 2.40(\mathrm{~s}, 3 \mathrm{H})$; $\delta_{\mathrm{C}}\left(75 \mathrm{MHz}, \mathrm{CDCl}_{3}\right) 160.7,156.2,137.6,137.0,135.7,129.2(2 \mathrm{C}), 128.6$ (2C), 126.9, 117.2, 116.7, 106.9, 102.0, 65.7, 55.5, 21.4; MS (APCI+) $m / z$ 253.0 $\left(\mathrm{MH}^{+}\right)$. Anal. Calcd for $\mathrm{C}_{17} \mathrm{H}_{16} \mathrm{O}_{2}$ : C, 80.93; H, 6.36, found: C, 80.74; $\mathrm{H}, 6.30$.

\section{5-(4-methoxycyclohexa-2,4-dien-1-yl)-2,3-dihydrobenzo[b]oxepine (4)}

Yield: 64\%; colorless oil; $\mathrm{R}_{\mathrm{f}}$ : 0.66 (EtOAc/c-hexane: $\left.2 / 8\right)$; IR $\left(\mathrm{v}, \mathrm{cm}^{-1}\right)$ 2933, 2238, 2163, 2012, 1608, 1511, 1487, 1442, 1248, 1180, 1036, 825, $760 ; \delta_{\mathrm{H}}\left(300 \mathrm{MHz}, \mathrm{CDC1}_{3}\right) 7.24-7.18(\mathrm{~m}, 3 \mathrm{H}), 7.10(\mathrm{~d}, 1 \mathrm{H}, J=7.8 \mathrm{~Hz})$, $7.03-6.98(\mathrm{~m}, 2 \mathrm{H}), 6.86(\mathrm{~d}, 2 \mathrm{H}, J=8.8 \mathrm{~Hz}), 6.26(\mathrm{t}, 1 \mathrm{H}, J=6.2 \mathrm{~Hz}), 4.50$ $(\mathrm{t}, 2 \mathrm{H}, J=6.2 \mathrm{~Hz}), 3.83(\mathrm{~s}, 3 \mathrm{H}), 2.47(\mathrm{q}, 2 \mathrm{H}, J=6.2 \mathrm{~Hz}) ; \delta_{\mathrm{C}}(75 \mathrm{MHz}$, $\left.\mathrm{CDCl}_{3}\right)$ 159.1, 158.0, 141.2, 135.4, 133.1, 131.3, $129.8(2 \mathrm{C}), 128.5,127.2$, 123.3, 122.0, 113.7 (2C), 78.0, 55.4, 30.0; MS (APCI+) $m / z 237.0\left(\mathrm{MH}^{+}\right)$. Anal. Calcd for $\mathrm{C}_{17} \mathrm{H}_{18} \mathrm{O}_{2}$ : C, 80.28; H, 7.13, found: C, 80.12; H, 6.97. 


\section{Acknowledgments}

The CNRS is gratefully acknowledged for financial support of this research. We thank the MNRES for a financial fellowship to E.R. and ICSN for a doctoral fellowship to B.T. Our laboratory BioCIS-UMR 8076 is a member of the Laboratory of Excellence LERMIT supported by a grant from ANR (ANR-10-LABX-33).

[1] Schweizer, E. E.; Meeder-Nycz, D. In Heterocyclic Compounds: Chromenes; Ellis, G. P., Ed.; Wiley: New York, 1977; pp 11-139.

[2] (a) Korec, R.; Sensch, K. H.; Zoukas, T. Arzneim. Forsch. 2000, 50 122. (b) Engler, T. A.; LaTessa, K. O.; Iyengar, R.; Chai, W., Agrios, K. Bioorg. Med. Chem. 1996, 4, 1755-1769. (c) Kashiwada, Y.; Yamazaki, K.; Ikeshiro, Y.; Yamagishi, T.; Fujioka, T.; Mihashi, K., Mizuki, K.; Cosentino, L. M.; Fowke, K.; Morris-Natschke, S. L.; Lee, K.-H. Tetrahedron 2001, 57, 1559-1563. (d) Kidway, M.; Saxena, S.; Khan, M. K. R.; Thukral, S. S. Bioorg. Med. Chem. Lett 2005, 15, 4295-4298. (e) Tahtaoui, C.; Demailly, A.; Guidemann, C.; Joyeux, C.; Schneider, P. J. Org. Chem. 2010, 75, 3781-3785. (f) Alami, M.; Peyrat, J.-F.; Belachmi, L.; Brion, J.-D. Eur. J. Org. Chem. 2001, 4207-4212. (g) Lago, J. H. G.; Ramos, C. S.; Casanova D. C. C.; Morendim, A. de A.; Bergamo, D. C. B.; Cavalheiro, A. J Bolzani, V. da S.; Furlan, M.; Guimaraes, E. F.; Young, M. C. M. Kato, M. J. J. Nat. Prod. 2004, 67, 1783-1788. (h) Cai, S. X.; Jiang, S.; Attardo, G.; Denis, R.; Storer, R.; Rej, R. PCT Int. Appl. WO 2003096982 A2 2003. (i) Li, S. R.; Chen, H. M.; Chen, P. Y.; Tsai, J. C.; Chen, L. Y.; Wang, E. C.; Huang, Y. T.; Wei, Y. C.; Lu, P. J. J. Chin. Chem. Soc. 2008, 55, 923-932.

[3] Teng, M.; Duong, T. T.; Johnson, A. T.; Klein, E. S.; Wang, L.; Khalifa, B.; Chandraratna, R. A. S. J. Med. Chem. 1997, 40, 24452451.

[4] Paciorek, P. M.; Burden, D. T.; Burke, Y. M.; Cowlrick, I. S.; Perkins, R. S.; Taylor, J. C.; Waterfall, J. F. J. Cardiovasc. Pharmacol. 1990, 15, 188-197.

[5] (a) Mousset, C.; Giraud, A.; Provot, O.; Hamze, A.; Bignon, J.; Liu, J. M.; Thoret, S.; Dubois, J.; Brion, J.-D.; Alami, M. Bioorg. Med. Chem. Lett. 2008, 18, 3266-3271; (b) Hamze, A.; Giraud, A.; Messaoudi, S.; Provot, O.; Peyrat, J.-F.; Bignon, J.; Liu, J. M.; Wdzieczak-Bakala, J.; Thoret, S.; Dubois, J.; Brion, J.-D.; Alami, M. ChemMedChem 2009, 4, 1912-1924. (c) Messaoudi, S.; Treguier, B.; Hamze, A.; Provot, O.; Peyrat, J.-F.; De Losada, J. R.; Liu, J. M.; Bignon, J.; Wdzieczak-Bakala, J.; Thoret, S.; Dubois, J.; Brion, J.D.; Alami, M. J. Med. Chem. 2009, 52, 4538-4542. (d) Messaoudi, S.; Hamze, A.; Provot, O.; Treguier, B.; Rodrigo De Losada, J.; Bignon, J.; Liu, J. M.; Wdzieczak-Bakala, J.; Thoret, S.; Dubois, J.; Brion, J. D.; Alami, M. ChemMedChem 2011, 6, 488-497. (e) Soussi, M. A.; Aprile, S.; Messaoudi, S.; Provot, O.; Del Grosso, E.; Bignon, J.; Dubois, J.; Brion, J. D.; Grosa, G.; Alami, M. ChemMedChem 2011, 6, 1781-1788. (f) Hamze, A.; Rasolofonjatavo, E.; Provot, O.; Mousset, C.; Veau, D.; Rodrigo, J.; Bignon, J.; Liu, J. M.; Wdzieczak-Bakala, J.; Thoret, S.; Dubois, J.; Brion, J. D.; Alami, M. ChemMedChem 2011, 6, 2179-2191. (g) L'Hermite, N.; Giraud, A.; Provot, O.; Peyrat, J.-F.; Alami, M.; Brion, J.-D. Tetrahedron 2006, 62, 11994-12002. (h) Mousset, C.; Provot, O.; Hamze, A.; Bignon, J.; Brion, J.-D.; Alami, M. Tetrahedron 2008, 64, 4287-4294. (i) Provot, O.; Giraud, A.; Peyrat, J.-F.; Alami, M.; Brion, J.-D. Tetrahedron Lett. 2005, 46, 8547-8550.

[6] (a) Eguchi, T.; Hoshino, Y.; Ayame, A. Bull. Chem. Soc. Jpn. 2002, 75, 581-585. (b) Kinoyama, I.; Miyamoto, S.; Miyazaki, T.; Koganemaru, Y.; Kawamoto, Y.; Shiraishi, N.; Hoshii, H.; Kuroda, A.; Yamazaki, M.; Yasuda, M.; Mizuno, H. Eur. Patent, 2010, EP $2177505 \mathrm{~A} 1$

[7] Donnelly, D. M. X.; Finet, J.-P.; Guiry, P. J.; Nesbitt, K. Tetrahedron 2001, 57, 413-423.

[8] Li, S.-R.; Chen, L.-Y.; Tsai, J.-C.; Tzeng, J.-Y.; Tsai, I.-L.; Wang, E.-C. Tetrahedron Lett. 2007, 48, 2139-2141.

[9] (a) Foroumadi, A.; Samzadeh-Kermani, A.; Emami, S.; Dehghan, G.; Sorkhi, M.; Arabsorkhi, F.; Heidari, M. R.; Abdollahi, M.; Shafiee, A. Bioorg. Med. Chem. Lett. 2007, 17, 6764-6769; (b) Mukerjee, S.
K.; Saroja, T.; Seshadri, T. R. Tetrahedron 1971, 27, 799-803; (c) Pinard, E.; Gaudry, M.; Hénot, F.; Thellend, A. Tetrahedron Lett 1998, 39, 2739-2742. (d) Whitehead, A.; Moore, J. D.; Hanson, P. R. Tetrahedron Lett. 2003, 44, 4275. (e) Worlikar, S.A.; Kesharwani, T.; Yao, T.; Larock, R. J. Org. Chem. 2007, 72, 1347-1353. (f) Commandeur, C.; Florent, J.-C.; Rousselle, P.; Bertounesque, E. Eur. J. Org. Chem. 2011, 1447-1451.

[10] Rasolofonjatovo, E.; Tréguier, B.; Provot, O.; Hamze, E.; Brion, J.D.; Alami, M. Tetrahedron Lett. 2011, 52, 1036-1040.

[11] (a) Rasolofonjatovo, E.; Provot, O.; Hamze, A.; Bignon, J.; Thoret, S.; Brion, J. D.; Alami, M. Eur. J. Med. Chem. 2010, 45, 3617-3626. (b) Ohsumi, K.; Nakagawa, R.; Fukuda, Y.; Hatanaka, T.; Morinaga, Y.; Nihei, Y.; Ohishi, K.; Suga, Y.; Akiyama, Y.; Tsuji, T. J. Med. Chem. 1998, 41, 3022-3032. (c) Liron, F.; Gervais, M.; Peyrat, J.-F. Alami, M.; Brion, J.-D. Tetrahedron Lett. 2003, 44, 2789-2794.

[12] (a) Sonogashira, K.; Tohda, Y.; Hagihara, N. Tetrahedron Lett. 1975 4467-4470; (b) Alami, M.; Ferri, F.; Linstrumelle, G. Tetrahedron Lett. 1993, 34, 6403-6406.

[13] (a) Olivi, N.; Thomas, E.; Peyrat, J.-F.; Alami, M.; Brion, J.-D Synlett 2004, 2175-2179; (b) Le Bras, G.; Provot, O.; Peyrat, J.-F.; Alami, M.; Brion, J.-D. Tetrahedron Lett. 2006, 47, 5497-5501; (c) Le Bras, G.; Hamze, A.; Messaoudi, S.; Provot, O.; Le Calvez, P. B.; Brion, J.-D.; Alami, M. Synthesis 2008, 1607-1611; (d) Jacubert, M. Hamze, A.; Provot, O.; Peyrat, J.-F.; Brion, J.-D.; Alami, M. Tetrahedron Lett. 2009, 50, 3588-3592; (e) Jacubert, M.; Provot, O.; Peyrat, J.-F.; Hamze, A.; Brion, J.-D.; Alami, M. Tetrahedron 2010, $66,3775-3787$.

[14] (a) For recent reviews, see: (a) Barluenga, J.; Valdes, C. Angew. Chem. Int. Ed. 2011, 50, 7486-7500. (b) Shao, Z.; Zhang, H. Chem. Soc. Rev. 2011, doi: 10.1039/C1CS15127D. For the coupling of $N$ tosylhydrazones with aryl halides or triflates, see: (c) Barluenga, J.; Moriel, P.; Valdes, C.; Aznar, F. Angew. Chem. Int. Ed. 2007, 46, 5587-5590. (d) Barluenga, J.; Tomas-Gamasa, M.; Moriel, P.; Amar, F.; Valdes, C. Chem. Eur. J. 2008, 14, 4792-4795. (e) Barluenga J.; Escribano M.; Moriel P.; Aznar F.; Valdés C. Chem. Eur. J. 2009, 15, 13291-13294. (f) Tréguier, B.; Hamze, A.; Provot, O.; Brion, J. D. Alami, M. Tetrahedron Lett. 2009, 50, 6549-6552. (g) Brachet, E.; Hamze, A.; Peyrat, J.-F.; Brion, J.-D.; Alami, M. Org. Lett. 2010, 12 4042-4045. (h) Barluenga, J.; Escribano, M.; Aznar, F.; Valdés, C. Angew. Chem. Int. Ed. 2010, 49, 6856-6859. (i) Barluenga, J.; Tomás-Gamasa, M.; Aznar, F.; Valdés, C. Chem. Eur. J. 2011, 16, 12801-12803. (j) Barluenga, J.; Florentino, L.; Aznar, F.; Valdés, C. Org. Lett. 2011, 13, 510-513.

[15] The $Z$-stereochemistry of all olefins 2 was determined by NOESY experiments on the crude mixture.

[16] Wadsworth, D. H.; Geer, S. M.; Detty, M. R. J. Org. Chem. 1987, 52, 3662-3668.

[17] Sawoo, S.; Srimani, D.; Dutta, P.; Lahiri, R.; Sarkar, A. Tetrahedron 2009, 65, 4367-4374

[18] Clair J. Collins,C.J.; Hanack, M.; Stutz, H.; Auchter, G.; Schoberth, W. J. Org. Chem. 1983, 48, 5260-5268.

[19] Hill, L. L.; Smith, J. M.; Brown, W. S.; Moore, L. R.; Guevera, P.; Pair, E. S.; Porter, J.; Chou, J.; Wolterman, C. J.; Craciun, R.; Dixon, D. A.; Shaughnessy, K. H. Tetrahedron 2008, 64, 6920-6934.

[20] Wang, S.; Wang, M.; Wang, B.; Li, P.; Yang, J. Tetrahedron 2011, 67, 4800-4806.

[21] Franks, M.A.; Schrader, E.A.; Pietsch, E.C.; Pennella, D.R.; Torti, S.V.; Welker, M.E. Bioorg. Med. Chem. 2005, 13, 2221-2233.

Received: ((will be filled in by the editorial staff)) Published online: ((will be filled in by the editorial staff)) 


\section{Entry for the Table of Contents}

\section{Layout 2:}

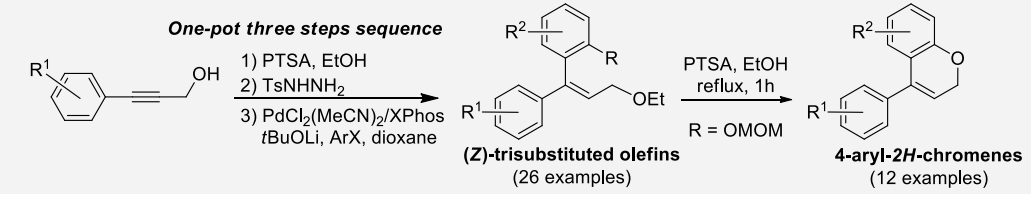

A one pot three-steps synthesis of (Z)-trisubstituted olefins from arylalkynols is

presented. Olefins having an ortho-OMOM substituent were successfully

transformed into 4-aryl-2H-chromenes using PTSA in EtOH in good to excellent yields.
(Heterocycle synthesis))

Evelia Rasolofonjatovo, Bret Tréguier, Olivier Provot,* Abdallah Hamze, JeanDaniel Brion and Mouâd Alami* Page No. - Page No.

A one pot three-steps synthesis of $Z$ trisubstituted olefins from arylalkynes and their cyclization into 4-aryl- $2 \mathrm{H}$-chromenes

Keywords: (Alkyne hydration/ Olefin / Chromene / $N$-tosylhydrazone/ Palladium) 
